\title{
Suppression of YAP by DDP disrupts colon tumor progression
}

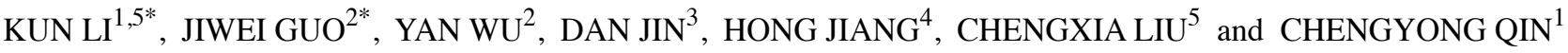 \\ ${ }^{1}$ Department of Gastroenterology, Shandong Provincial Hospital Affiliated to Shandong University, \\ Jinan, Shandong 250021; ${ }^{2}$ Cancer Research Institute; Departments of ${ }^{3}$ Pain Management, ${ }^{4}$ Anorectal Surgery \\ and ${ }^{5}$ Gastroenterology, Binzhou Medical University Hospital, Binzhou, Shandong 256603, P.R. China
}

Received September 25, 2017; Accepted February 28, 2018

DOI: $10.3892 /$ or.2018.6297

\begin{abstract}
Colon cancer is a commonly diagnosed cancer that often has a poor prognosis. Combined with the development of drug resistance to cancer treatment agents the treatment efficacy of colon cancer can be limited. Activation of the oncogene $Y A P$ has been shown to be related to colon cancer progression and is associated with poor prognosis, drug resistance and metastasis, even under treatment. Cisplatin (DDP) is a commonly used drug that can control carcinoma progression, although its mechanisms are poorly understood. In the present study, we examined whether DDP specifically suppressed YAP in order to inhibit colon carcinoma progression. Our data revealed that Mst/Yap signaling was unusually activated in colon cancers, promoting cell proliferation and invasion. DDP treatment decreased the expression of YAP at both the transcriptional and post-translational levels, leading to cell cycle arrest, apoptosis and senescence in cancer cells in addition to decreasing epithelial-to-mesenchymal transition, cell motility and in vitro cell inyasion and migration Ultimately, DDP increased the expression of E-cadherin and decreased the expression of vimentin. The present study also revealed that post-translational regulation of YAP phosphorylation controlled the subcellular distribution between the nucleus and the cytoplasm. In conclusion, the findings of the present study revealed that DDP was a suitable therapeutic candidate for colon cancer that specifically targets the Mst/Yap signaling pathway.

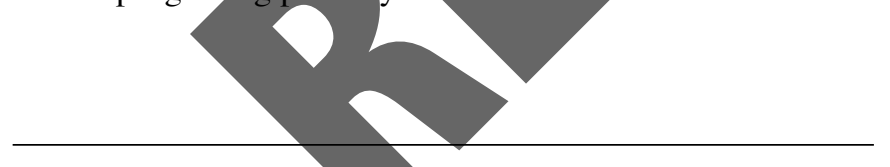

Correspondence to: Dr Chengxia Liu, Department of Gastroenterology, Binzhou Medical University Hospital, 661 Huanghe Second Road, Binzhou, Shandong 256603, P.R. China

E-mail:phdlcx@163.com

Dr Chengyong Qin, Department of Gastroenterology, Shandong Provincial Hospital Affiliated to Shandong University, 324 Jingwu Road, Jinan, Shandong 250021, P.R. China

E-mail:ddwuyou@126.com

${ }^{*}$ Contributed equally

Key words: DDP, YAP, colon cancer, epithelial-mesenchymal transition

\section{Introduction}

Colon cancer is one of the most frequently diagnosed cancers worldwide and has a particularly poor prognosis for patients (1). Despite the development of some novel-targeted therapies, chemotherapy to inhibit and damage cancer cells using chemicals such as cisplatinum remains one of the most common treatment options for colon cancer. However, chemotherapy is often indiscriminate, with many off-targeted toxic effects and is typically only used to treat colon cancers in the early stages of the disease. During this period, cancer cells often acquire drug resistance due to increased exposure to stressful conditions and cytotoxic drugs (2). This resistance is an important obstacle to treatment that can result in chemotherapy failure in many cancers, including colon cancer (3). Therefore, the identification of safe and effective agents that specifically target drug-resistant cancers, is vital for the future treatment of colon cancer (4-6).

Due to the inevitable and often rapid development of drug resistance to colon cancer chemotherapies, there has been some research investigating the possible underlying mechanisms. For example, the aberrant activation of the Mst/Yap-signaling pathway has been revealed to correlate with drug tolerance acquired during the treatment of colon cancer patients using cisplatin (DDP) and erlotinib, an epidermal growth factor receptor (EGFR) inhibitor (7). However, the role of the Mst/Yap pathway in the development of drug resistance is poorly understood in human colon cancers. Functionally, the Mst/Yap pathway is highly conserved in both humans and Drosophila and has a key role in regulating growth $(8,9)$. The tumor suppressor mercaptopyruvate sulfurtransferase (MPST or MST) and a subsequent kinase cascade, act to negatively regulate YAP, an oncoprotein involved in cell growth and survival that functions by transcriptionally regulating various downstream target genes (10). MST is also one of the core suppressor molecules in the Hippo signaling pathway and is phosphorylated and activated by various upstream signaling proteins. Salvador family WW domain-containing protein 1 (SAV1 or WW-45) is another core component of the Hippo signaling pathway and activated MST combines with SAV1 to phosphorylate and activate the large tumor suppressor 1 (LATS1) kinase. Activated LATS1 binds with the MOB kinase activator MOB1 to phosphorylate YAP and this phosphorylated protein is retained in the cytoplasm through interactions with the 14-3-3 family of proteins. By 
preventing movement to the nucleus, YAP is prevented from combining with other transcription factors to inactivate target promoters (11-14). However, without the suppressive functions of MST, unphosphorylated YAP gathers in the nucleus and interacts with transcriptional enhancer factor domain (TEAD) transcription factors. This in turn regulates the Mst/Yap pathway via downstream genes that include cysteine rich angiogenic inducer 61 (CYR61), connective tissue growth factor (CTGF), survivin (BIRC5) and cyclin D1 (CCND1) (15-18).

The chemotherapeutic agent DDP is one of the most extensively used agents for the treatment of cancer. In 1972, it became the first metal-based drug to enter clinical trials and was initially applied in a clinical setting in 1979 (19). DDP is now a gold standard drug used for the treatment of testicular cancer (for which it has a $90 \%$ cure rate) and also for the treatment of head and neck, cervical, breast, lung, ovarian, gastric and bladder cancers, among many others $(20,21)$. DDP exerts its antitumor activity through its alkylating properties. Once the drug enters the cytoplasm of a cell, chloride ligands are spontaneously and sequentially replaced with water molecules due to the fact that the chloride concentration of the cytoplasm is much lower than that of the blood. This results in the formation of positively charged bis-aquated platinum complexes that bind to DNA (22-25). DDP predominantly forms intra-strand adducts between two adjacent guanines that are followed by an adjacent guanine and adenine. These adducts cause the DNA helix to bend by up to $60 \%$ towards the major groove and unwind, inhibiting further DNA replication and transcription. This ultimately leads to cell death $(21,26,27)$. Howeve the continuing clinical success of DDP is hindered by two major limitations, the development of DDP-resistant cance cells and the toxic side-effects of the drug.These mechanisms act in tandem, so that when cells become resistant to DDP, the subsequent dose must be increased. This in turn increases the severity of toxic side-effects. These side-effects are primarily due to the dose-limiting effects of the drug on neurotoxicity and ototoxicity, although other common side-effects include severe nausea, vomiting, gastrotoxicity and myelosuppression (28-31).

To further investigate the role of YAP in cancer drug resistance and to yalidate DDP as a colon cancer therapy, we examined how DDP suppresses the Mst/Yap signaling pathway and the mechanism through which this leads to the inhibition of colon carcinoma progression and metastasis. Our data demonstrated that DDP specifically suppressed the expression of YAP and had various downstream effects on transcription. In addition, we confirmed that DDP has the potential to be used as a treatment for colon cancer. These data improved our understanding of the Mst/Yap pathway and its role in cancer progression and may lead to improved outcomes for patients.

\section{Materials and methods}

Molecular biology. Flag-tagged YAP constructs were made using the pcDNA 3.1 vector (Invitrogen, Carlsbad, CA, USA). Sequences encoding the Flag epitope (DYKDDDDK) were added by PCR through the replacement of the first Met-encoding codon in the respective cDNA clones.

Cell culture and transient transfection. Human colon cancer cells SW620, LoVo and NCM460 obtained from the American
Type Culture Collection (ATCC; Manassas, VA, USA) were cultured in Dulbecco's modified Eagle's medium (DMEM) supplemented with $10 \%$ fetal bovine serum (FBS; Hyclone Laboratories, San Angelo, TX, USA) in an incubator, at $37^{\circ} \mathrm{C}$ under a mixture of $95 \%$ air and $5 \% \mathrm{CO}_{2}$. Plasmids were transfected using polyethylenimine (PEI) reagent, following the manufacturer's instructions.

Western blot analysis. SW620 and LoVo colon cancer cells were transfected with the relevant plasmids and cultured for $36 \mathrm{~h}$. For western blot analysis, the cells were lysed in NP-40 buffer $(10 \mathrm{mM}$ Tris $\mathrm{pH} 7.4,150 \mathrm{mM} \mathrm{NaCl}, 1 \%$ Triton $\mathrm{X}-100$, $1 \mathrm{mM}$ EDTA pH 8.0, $1 \mathrm{mM}$ EGTA pH 8.0, $1 \mathrm{mM}$ PMSF and $0.5 \% \mathrm{NP}-40$ ) at $25^{\circ} \mathrm{C}$ for $40 \mathrm{~min}$. The lysates were added to $5 \mathrm{X}$ loading dye and then separated by electrophoresis.

Antibodies. The primary antibodies used in the present study were rabbit anti-Flag (1:1,000, cat. no. sc-166355; Santa Cruz Biotechnology, Inc., Dallas, TX, USA), antipYap (cat. no. 14074) and anti-Yap (cat no. 13008) (1:1,000; Cell Signaling Technology, Inc., Danvers, MA, USA), antivimentin (cat. no. ab92547), anti-E-cadherin (cat. no. ab1416) and anti-cleaved capase-3 (1:1,000, cat. no. ab2302; all from Abcam, Cambridge, UK).

Immunofluorescent staining. To examine the subcellular localization of YAP, the SW620 cells were exposed to DDP for $36 \mathrm{~h}$, seeded onto coverslips in a 24-well plate, and left overnight. The cells were then fixed using $4 \%$ formaldehyde for $30 \mathrm{~min}$ at $25^{\circ} \mathrm{C}$ and treated with $2 \%$ bovine serum albumin (BSA) in phosphate-buffered saline (PBS) for $30 \mathrm{~min}$. The coverslips were incubated with rabbit anti-YAP monoclonal antibody (Cell Signaling Technology, Inc.) at 1:200 dilution in 3\% BSA. The coverslips were then incubated with an Alexa Fluor 594 (red, cat. no. R37117; Invitrogen; Thermo Fisher Scientific, Inc., Waltham, MA, USA) tagged anti-rabbit monoclonal secondary antibody at $1: 1,000$ dilution in $3 \%$ BSA. DAPI $(3 \mu \mathrm{g} / \mathrm{ml})$ was added for nuclear counterstaining. Images were captured with a Zeiss Axio Imager Z1 fluorescence microscope (Carl Zeiss AG, Oberkochen, Germany).

Cell flow cytometry assays. DDP-treated and control cells at $80 \%$ confluency were harvested and fixed with $70 \%$ ethanol. These were then stained using propidium iodide (PI) and the cell cycle stage was assessed by flow cytometry. To evaluate apoptosis, the cells were cultured at $80 \%$ confluency, trypsinized and stained with a PI/Annexin V Apoptosis Detection kit (Vazyme, Jiangsu Sheng, China). The data were collected and analyzed on a BD FACScan flow cytometer using FACS software (BD Biosciences, San Jose, CA, USA).

RNA isolation and real-time reverse transcription ( $R T)-P C R$ assay. We used TRIzol reagent (TransGen Biotech, Beijing, China) to isolate total RNA from the samples. RNA was reverse transcribed into first-strand cDNA using a TransScript All-in-One First-Strand cDNA Synthesis kit (TransGen Biotech). cDNAs were used in a real-time RT-PCR assay with the human $G A P D H$ gene as an internal control. The RT-PCR primers were: E-cadherin forward, ACCATTAACAGGAAC ACAGG and reverse, CAGTCACTTTCAGTGTGGTG; YAP 
forward, GGACCCCAGACGACTTCCTCAACAG and reverse, CCTTCCAGTGTGCCAAGGTCCACAT; CYR61 forward, GGTCAAAGTTACCGGGCAGT and reverse, GGA GGCATCGAATCCCAGC; CTGF forward, AATGCTGCGA GGAGTGGGT and reverse, CGGCTCTAATCATAGTTGG GTCT; vimentin forward, CGCCAACTACATCGACAA GGTGC and reverse, CTGGTCCACCTGCCGGCGCAG.

SA- $\beta$-gal staining. Senescent cells were detected using a Senescence $\beta$-Galactosidase Staining kit (Beyotime Institute of Biotechnology, Jiangsu, China), according to the manufacturer's instructions.

Cell Counting Kit-8 (CCK-8) analysis. For CCK-8 analysis, $100 \mu \mathrm{l}$ of SW620 cell suspensions were added to a 96-well plate $(5,000$ cells/well). The plates were pre-incubated for $24 \mathrm{~h}$ in a humidified incubator at $37^{\circ} \mathrm{C}$ under $5 \% \mathrm{CO}_{2}$. Subsequently, $10 \mu \mathrm{l}$ of the test treatments were added to the test plates. The plates were then incubated for a further $12 \mathrm{~h}$ under the same incubation conditions. Subsequently, $10 \mu$ l of CCK-8 solution was added to each well and then the plates were incubated for $4 \mathrm{~h}$. Finally, the absorbance at $450 \mathrm{~nm}$ was assessed using a microplate reader.

Human colon cancer specimen collection. All human colon cancer and normal colon tissue specimens were collected from the Affiliated Hospital of Binzhou Medical College. The experimental protocol was approved by the Research Ethics Committee of Binzhou Medical University (approval no. 2017-014-01 for the human tissues and 2017-05-02 for the mouse tissues). Written informed consents were obtained from all patients. A total of nine human colon tumor samples with matched normal colon samples yere used for RT-PCR analysis. Eight colon cancer samples were used for western blot analysis.

Analysis of publicly available datasets. To analyze the correlation between the expression level of YAP and the prognostic outcome of patients, Kaplan-Meier survival curves of colon tumor patients with low and high expression of YAP were generated using Kaplan-Meier plotter (www.kmplot.com/analysis).

Wound healing assays. To assess the effects of DDP on wound healing, $10^{5}$ cells were seeded onto 6-well plates and left for 1 day before treatment with DDP. These cells were then incubated in $5 \% \mathrm{CO}_{2}$ at $37^{\circ} \mathrm{C}$ for $24 \mathrm{~h}$. A wound was scraped into the cells using a plastic 200- $\mu$ l pipette tip and then washed by PBS. The cells were incubated in DMEM containing 10\% FBS with $15 \mu \mathrm{g} / \mathrm{ml}$ of DDP and various plasmids at different time-points to assess wound healing.

Transwell migration assays. Transwell migration assays were performed using a 24-well chamber (Costar 3422; Corning Inc., Corning, NY, USA). The lower and upper chambers were separated by a polycarbonate membrane $(8-\mu \mathrm{m}$ pore size). SW620 cells $\left(1 \times 10^{5}\right)$ were seeded into DMEM without FBS in the upper chamber. DMEM containing $10 \%$ FBS was added to the lower chamber. The cells were allowed to migrate for $36 \mathrm{~h}$ at $37^{\circ} \mathrm{C}$ in a humidified atmosphere containing $5 \% \mathrm{CO}_{2}$. The cells remaining on the upper side of the membrane were removed using PBS-soaked cotton swabs. The membrane was then fixed in $4 \%$ paraformaldehyde for $20 \mathrm{~min}$ at $37^{\circ} \mathrm{C}$ and then stained with crystal violet. The cells on the lower side of the membrane were counted under an Olympus light microscope (Olympus, Tokyo, Japan).

MTT and CCK-8 assays. Cell viability was determined using 3-(4,5-dimethylthiazol-2yl)-2,5-diphenyltetrazolium bromide (MTT) and CCK-8 assays in 96-well plates in a manner similar to that above described for the CCK- 8 assays. The cells were incubated with various concentrations of DDP for $48 \mathrm{~h}$, followed by incubation with MTT and CCK- 8 for $4 \mathrm{~h}$. Subsequently, $100 \mu \mathrm{l}$ isopropanol (in $0.04 \mathrm{~N} \mathrm{HCL}$ ) was added to dissolve the formazan crystals for the MTT assay. Absorbance was read at $570 \mathrm{~nm}$ using a spectrophotometer (Tecan Group Ltd., Männedorf, Switzerland). The cell viability was assessed as relative absorbance compared to a dimethyl sulfoxide (DMSO)-only control.

In vivo experiments. To assess the in vivo effects of DDP, 3- to 5 -week old female BALB/c athynic (NU/NU) nude mice were housed in a level 2 biosafety laboratory and raised according to the Institutional Animal Gurdelines of Binzhou Medical University. All animal experiments were carried out with the prior approval of the Binzhou Medical University Committee on Animal Care. For the experiments, the mice were injected with $5 \times 10^{6} \mathrm{SW} 620$ cells and randomly divided into two groups (five mice per group) after the diameter of the xenograft tumors had reached $\sim 5 \mathrm{~mm}$. Xenograft mice were then intraperitoneally administered either DDP or PBS three times a week and tumor volume and body weight were assessed every second day. Tumor volume was estimated as $0.5 \mathrm{x} \mathrm{a}^{2} \mathrm{x}$ b (where $\mathrm{a}$ and $\mathrm{b}$ represent tumor short and long diameter, respectively). The mice were euthanized after 6 weeks of treatment and the tumors were assessed a final time. Subsequently, tumor and organ tissues were collected from xenograft mice and analyzed by immunohistochemistry.

Immunohistochemical analysis. Tumor tissues were fixed in $4 \%$ paraformaldehyde overnight and then embedded in paraffin wax. Four-micrometer thick sections were and stained using hematoxylin and eosin (H\&E) for histological analysis.

Statistical analysis. Data were analyzed using GraphPad Prism 5 (GraphPad Software Inc., La Jolla, CA, USA) and are presented as the means \pm SD. Two-tailed Student's t-tests were used to compare two groups and ANOVA with Tukey's posthoc test was used to compare multiple groups. P-value $<0.05$ was considered to indicate a statistically significant difference which is highlighted in the figures with an asterisk, while P-values $<0.01$ are highlighted using two asterisks and P-values $<0.001$ are highlighted using three asterisks.

\section{Results}

Abnormal activation of YAP in colon cancer tissues. To determine the expression of YAP in human colon cancer tissues, we analysed the demographic and tumor characteristics of 46 patients that met the inclusion criteria for participating in the study (Table I). Subsequently, we performed both RT-PCR 
and western blot analysis. This demonstrated that YAP mRNA levels were higher in the nine colon cancer tissues than in matched normal tissue (Fig. 1A). As displayed in Fig. 1B, the protein levels of YAP were also higher in eight colon cancer tissues histologically examined compared to adjacent normal tissue. Publicly available datasets (http://www.kmplot.com/ analysis/index.php? $\mathrm{p}=$ service $\&$ cancer $=$ gastric and colon) (32) were filtered and used to analyze the prognostic correlation between the survival of colon cancer patients and the expression of YAP. Kaplan-Meier analyses revealed that YAP protein levels had an inverse correlation with survival, with high expression associating with shorter overall survival (OS) $\left(\mathrm{n}=1926, \mathrm{P}=2.2 \times 10^{-6}\right)$ (Fig. 1C). The expression of YAP was also higher in SW620 and LoVo colon cancer cell lines relative to a normal NCM460 cell line. In addition, the amount of activated YAP found in the nucleus was higher in LoVo and SW620 colon cancer cells, while inactivated cytoplasmic YAP (p-YAP) was more abundant in normal NCM460 cells. Furthermore, the activated forms of YAP, the nuclear localization of YAP, was higher in LoVo and SW620 cells compared to normal NCM460 cells and the non-activated cytoplasmic localization form of YAP and p-YAP, was higher in normal NCM460 cells (Fig. 1D).

Downregulation of YAP inhibits cell growth and invasion. Both YAP specific silencing by siRNAs, and stable ectopic overexpression of YAP, were used to establish whether the activation of the oncogene affected the initiation, progression and metastasis of colon cancer (Fig. 2A and B). Knockdown of YAP was found to decrease cell growth (Fig. 2C), Ki-67 protein levels (Fig. 2D), clonal formation (Fig. 2E) and cell migration (Fig. 2F) in SW620 cells. The opposite effect was observed for each of these factors in SW620 cells that overexpressed ectopic YAP (Fig. 2C-F). Notably, depletion of YAP decreased the invasiveness of SW620 colon cancer cells (Fig. 2G), whereas overexpression increased cell invasion. These data demonstrated that YAP played an important role in colon cancer cell growth and invasion.

DDP suppresses YAP activity in colon cancer cells. To further investigate the mechanisms of DDP, we explored whether the drug affected the Mst/Yap signaling pathway in colon cancer cells. Our data revealed that DDP dosedependently (Fig. 3A) and time-dependently (Fig. 3B) reduced $Y A P$ mRNA and YAP protein levels in SW620 cells. Subsequently, we assessed if this was due to a direct effect of DDP on the promoter region of YAP. A luciferase reporter gene assay using the promoter region of YAP $(-1420 /+115$, pGL3-Yap) demonstrated that DDP decreased the luciferase activity of the promoter in a dose-dependent manner in both SW620 and LoVo cancer cells (Fig. 3C). This indicated that DDP downregulated the expression of $Y A P$ at the transcriptional level in colon cancer cells through a direct interaction. As DDP reduced the expression of $Y A P$ in colon cancer cells, we subsequently explored the effects on downstream target genes in the Mst/Yap signaling pathway. Both RT-PCR and western blot analysis revealed that treatment with $10 \mu \mathrm{g} / \mathrm{ml}$ of DDP for $72 \mathrm{~h}$ suppressed $Y A P$ and the downstream target genes CYR61 and CTGF mRNA in SW620 cells (Fig. 3D). Furthermore, semi-quantitative analysis of CYR61 and CTGF
Table I. Patients' demographics and tumor characteristics.

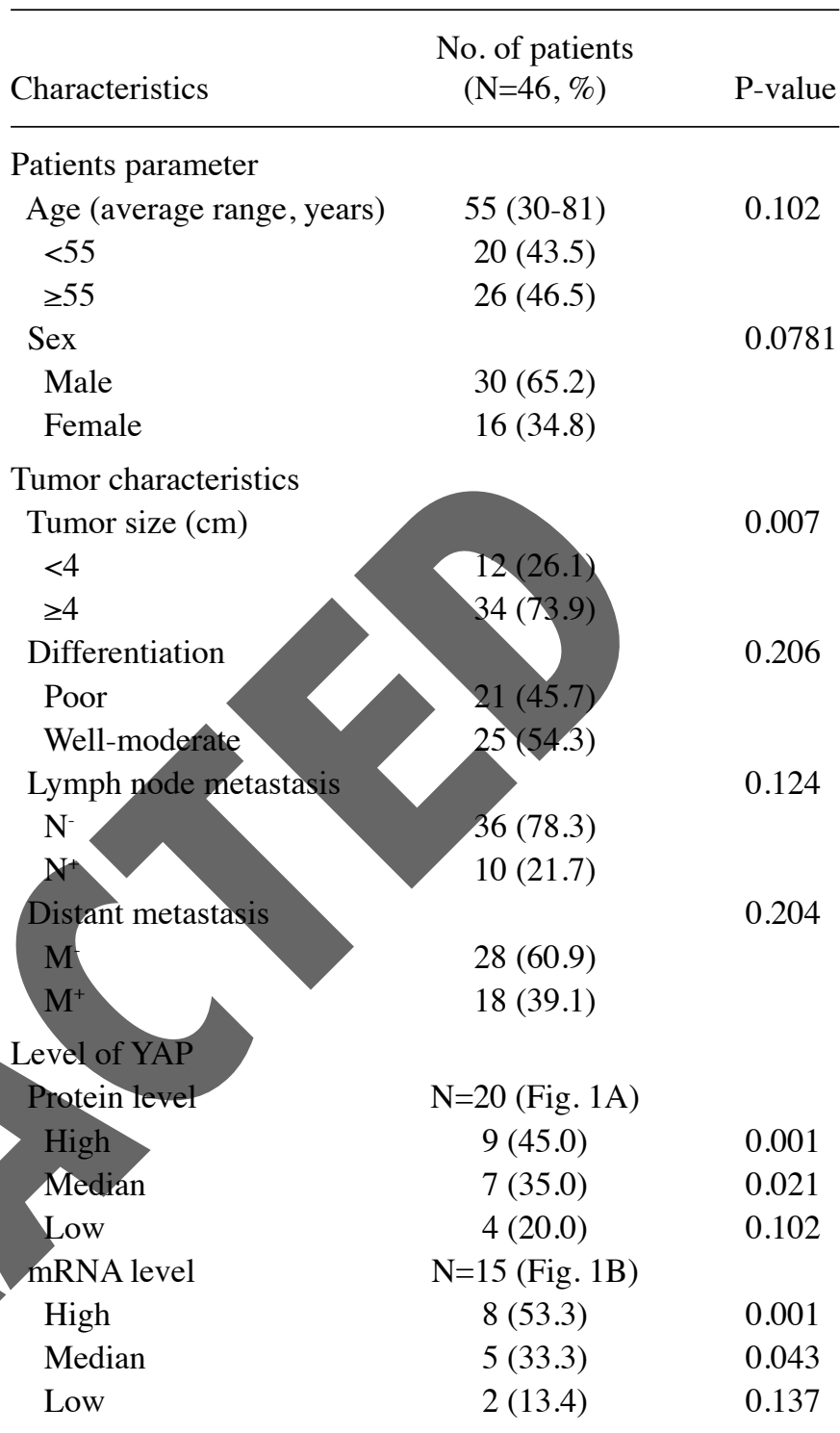

Association of the YAP level with clinicopathological features in colon tumor population. Data represent the mean \pm SD.

immunofluorescent staining indicated that treatment with DDP at $10 \mu \mathrm{g} / \mathrm{ml}$ for $72 \mathrm{~h}$ also decreased the expression of these proteins in SW620 cells (Fig. 3E). Our data also revealed that the levels of YAP protein were decreased in the nucleus after DDP treatment. Exposure to $6 \mu \mathrm{g} / \mathrm{ml} \mathrm{DDP}$ for $36 \mathrm{~h}$ increased the levels of phosphorylated YAP (p-YAP) in the cytoplasm of SW620 cells, leading to the translocation of YAP from the nucleus to the cytoplasm (Fig. 3F and G).

DDP affects YAP-mediated colon cell proliferation. Cell proliferation assays indicated that abnormally high expression of YAP increased the in vitro growth of SW620 and LoVo cells (Fig. 4A and B). Treatment with $10 \mu \mathrm{g} / \mathrm{ml}$ DDP showed a time-dependent arrest in the cellular growth of SW620 and LoVo cells, with or without the stable overexpression of YAP. Furthermore, semi-quantitative analysis of Ki-67 immunofluorescent staining indicated that $10 \mu \mathrm{g} / \mathrm{ml}$ DDP treatment for $72 \mathrm{~h}$ significantly reduced Ki-67 expression in SW620 
A

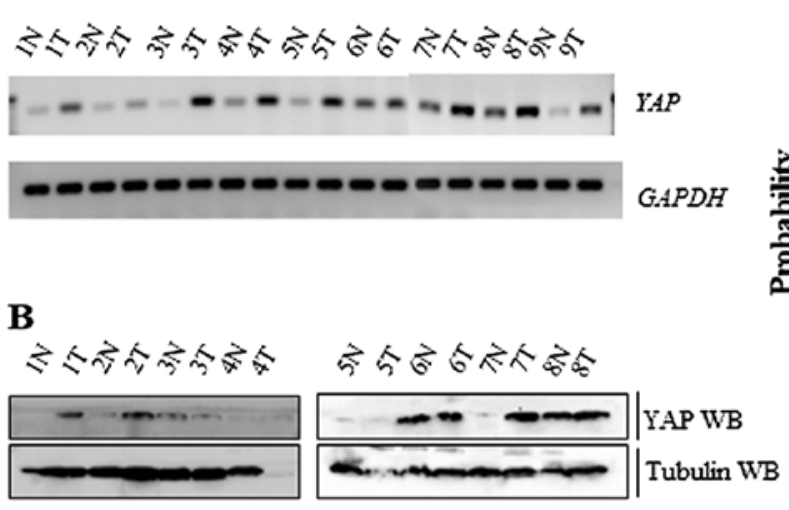

C

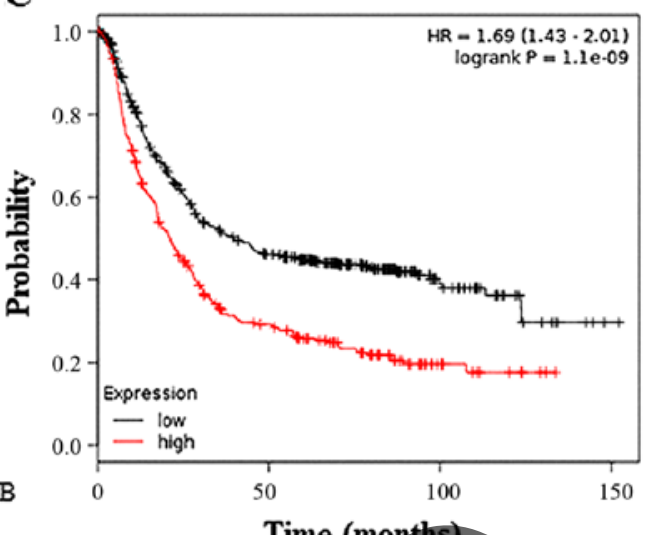

D

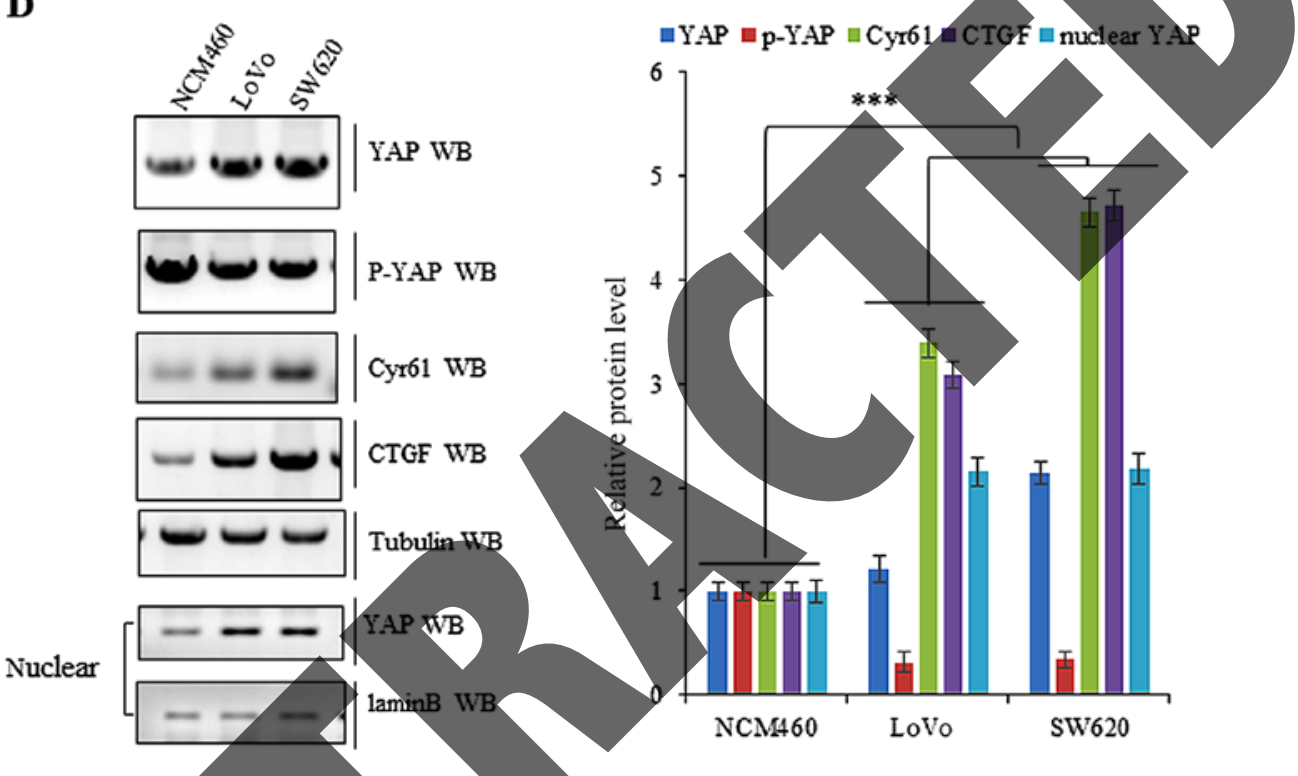

Figure 1. Aberrant activation of YAP in colon tumors from patients. (A) RT-PCR assays demonstrating increased YAP mRNA expression in human colon tissues compared to adjacent normal tissues. (B) Western blot analysis indicated that YAP protein levels were also increased in human colon tissues compared to adjacent normal tissues. (C) Kaplan-Meier OS curves for YAP expression ( $\mathrm{n}=1926, \mathrm{P}=1 \mathrm{E}-09$ using a log-rank test of significance). (D) Immunofluorescence of stained YAP demonstrating increased total protein levels, as well as increased nuclear YAP localization, in both LoVo and SW620 colon cancer cells relative to a normal NCM460 cell line. This associates with decreased p-YAP expression. Results were presented as mean \pm SD, and the error bars represent the SD of three independent experiments. " $\mathrm{P}<0.001$ vs. control group. OS, overall survival.<smiles>C1CCCC1</smiles>

cells (Fig. 4C). Colony formation assays using SW620 cells also revealed that ectopic overexpression of YAP reversed colony formation after treatment with DDP at $10 \mu \mathrm{g} / \mathrm{ml}$ for $72 \mathrm{~h}$ (Fig. 4D).

To fully explore the putative biological functions of DDP, cell cycle analysis in colon cancer cells under DDP treatment was performed. This revealed that treatment with $10 \mu \mathrm{g} / \mathrm{ml}$ DDP for $72 \mathrm{~h}$ induced cell cycle arrest in $\mathrm{G} 2$ phase and also blocked YAP-induced progression into $\mathrm{S}$ phase in SW620 cells. This was demonstrated using both quantitative analysis and representative histograms summarizing cell cycle distribution (Fig. 5A). Cell cycle and Annexin V flow cytometry also revealed that treatment with $10 \mu \mathrm{g} / \mathrm{ml}$ DDP for $72 \mathrm{~h}$ induced increased apoptosis in SW620 cells, whereas ectopic overexpression of YAP partially reduced DDP-induced apoptosis (Fig. 5B). Additional immunofluorescent staining of cleaved caspase-3 (a marker of apoptosis) confirmed that DDP increased the levels of this protein product. In addition, ectopic overexpression of YAP partially reduced DDP-induced apoptosis in SW620 cells over the 72-h period (Fig. 5C and D).

DDP affects YAP-mediated colon tumor cell senescence, invasiveness and epithelial-mesenchymal transition (EMT). Cellular apoptosis and senescence resistance is a common mechanism by which cancer cells avoid death. It has also been previously reported that aberrant activation of YAP inhibits senescence in human tumor cells (7). To analyze whether DDP influences YAP-induced cellular senescence in colon tumor cells, we used $\beta$-galactosidase staining to identify cells that were senescent. This indicated that exposure to $10 \mu \mathrm{g} / \mathrm{ml}$ DDP for $72 \mathrm{~h}$ increased the number of SW620 cells displaying senescence. Ectopic overexpression of YAP lowered the number of DDP-induced senescent cells, whereas knockdown of YAP using siRNA promoted senescence (Fig. 6A). Phase contrast 
$\mathbf{A}$

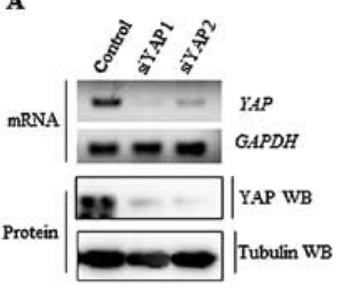

B

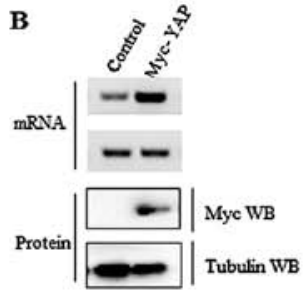

Tubulin WB
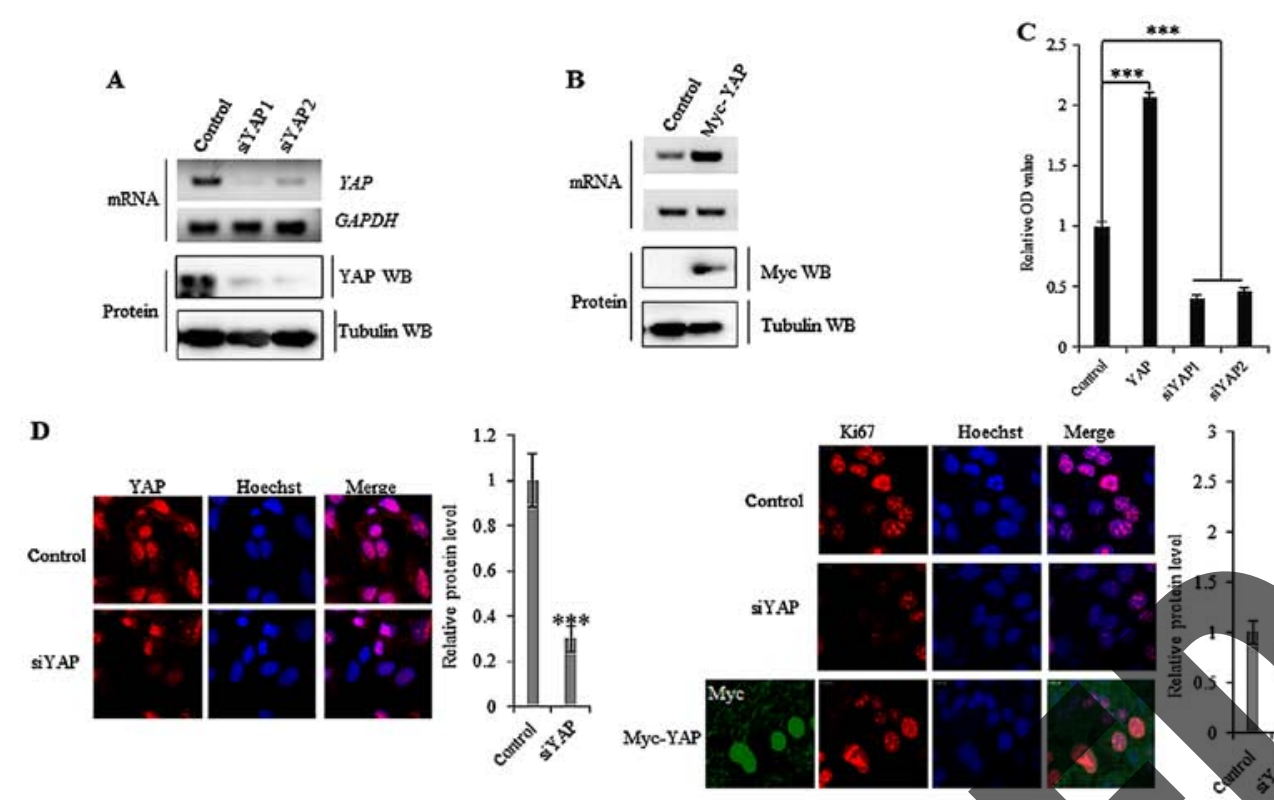
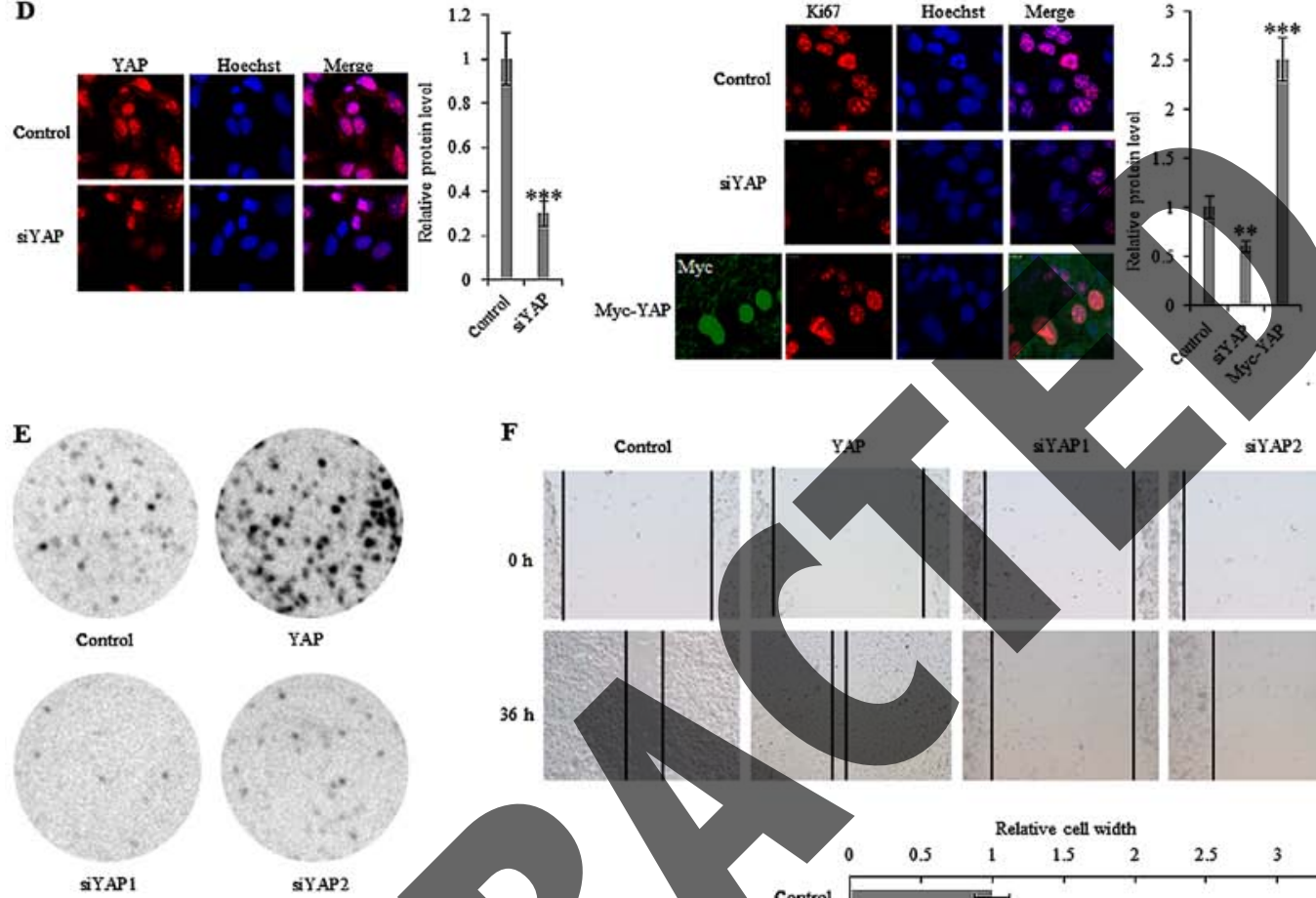

F

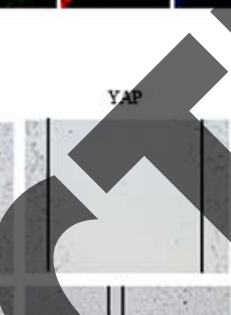

YAP

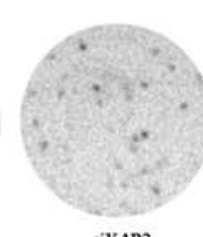

SIYAP2

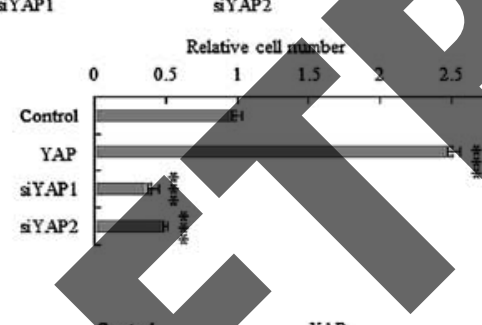

G
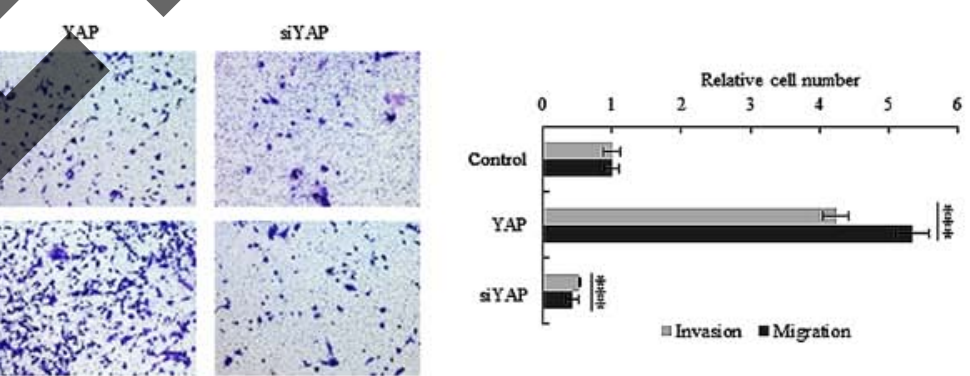

Figure 2. YAP knockdown inhibits cell growth and invasion. (A) RT-PCR and western blot analysis of the mRNA and protein expression levels of YAP after exposure to a YAP-specific shRNA in SW620 cells compared to control cells. (B) RT-PCR and western blot analysis of YAP mRNA and YAP protein levels after stable overexpression of YAP in SW620 cells compared to control cells. (C) In vitro proliferation was promoted in YAP overexpressing SW620 cells, whereas it was decreased in YAP knockdown cells. (D) Immunofluorescent staining of YAP and Ki-67 decreased after knockdown of YAP using a YAPspecific shRNA in SW620 cells compared to control cells. (E) Colony formation assays indicated that colony formation increased in YAP overexpressing cells and decreased in YAP knockdown cells. (F) Scratch assays showing that stably overexpressing YAP cells exhibited increased cell migration in SW620 cells after $36 \mathrm{~h}$, while YAP knockdown cells exhibited decreased cell migration. (G) Transwell assays demonstrated that YAP overexpressing cells showed increased invasion and migration in SW620 cells compared to control cells, while knockdown of YAP leads to decreased invasion and migration. Results were presented as mean $\pm \mathrm{SD}$, and the error bars represent the $\mathrm{SD}$ of three independent experiments. ${ }^{* *} \mathrm{P}<0.01{ }^{* * * *} \mathrm{P}<0.001 \mathrm{vs}$. control group.

microscopy analysis indicated that cellular morphology was markedly altered after treatment with $10 \mu \mathrm{g} / \mathrm{ml}$ DDP for $72 \mathrm{~h}$ in cells overexpressing YAP and in YAP-knockdown cells (Fig. 6B). 
A
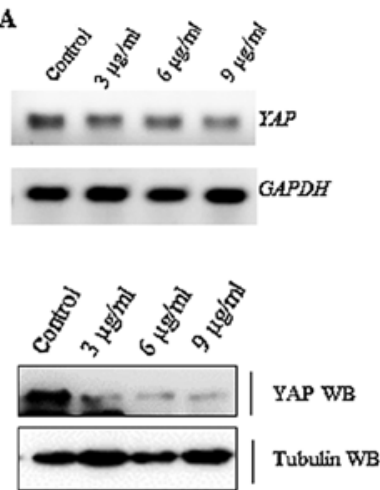

B
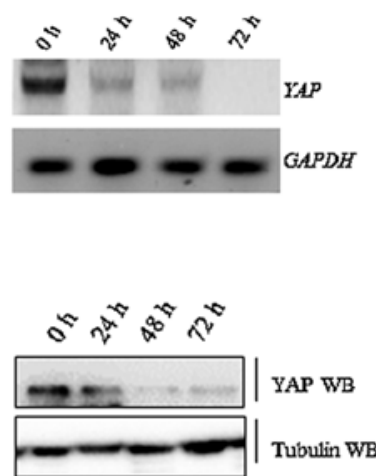

C

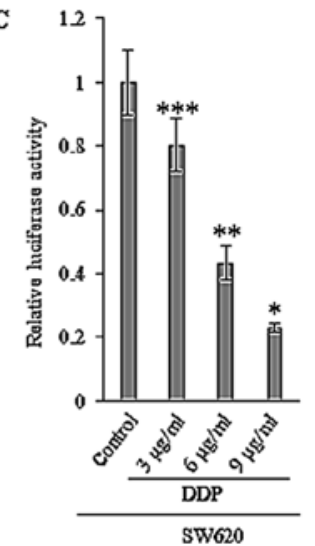

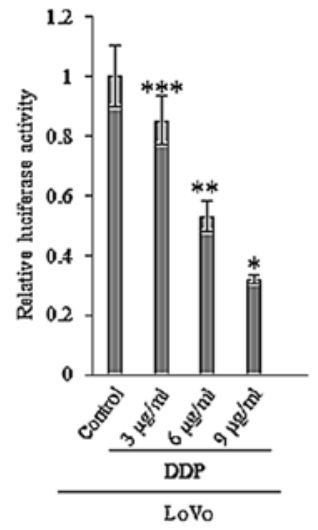

$\mathbf{D}$
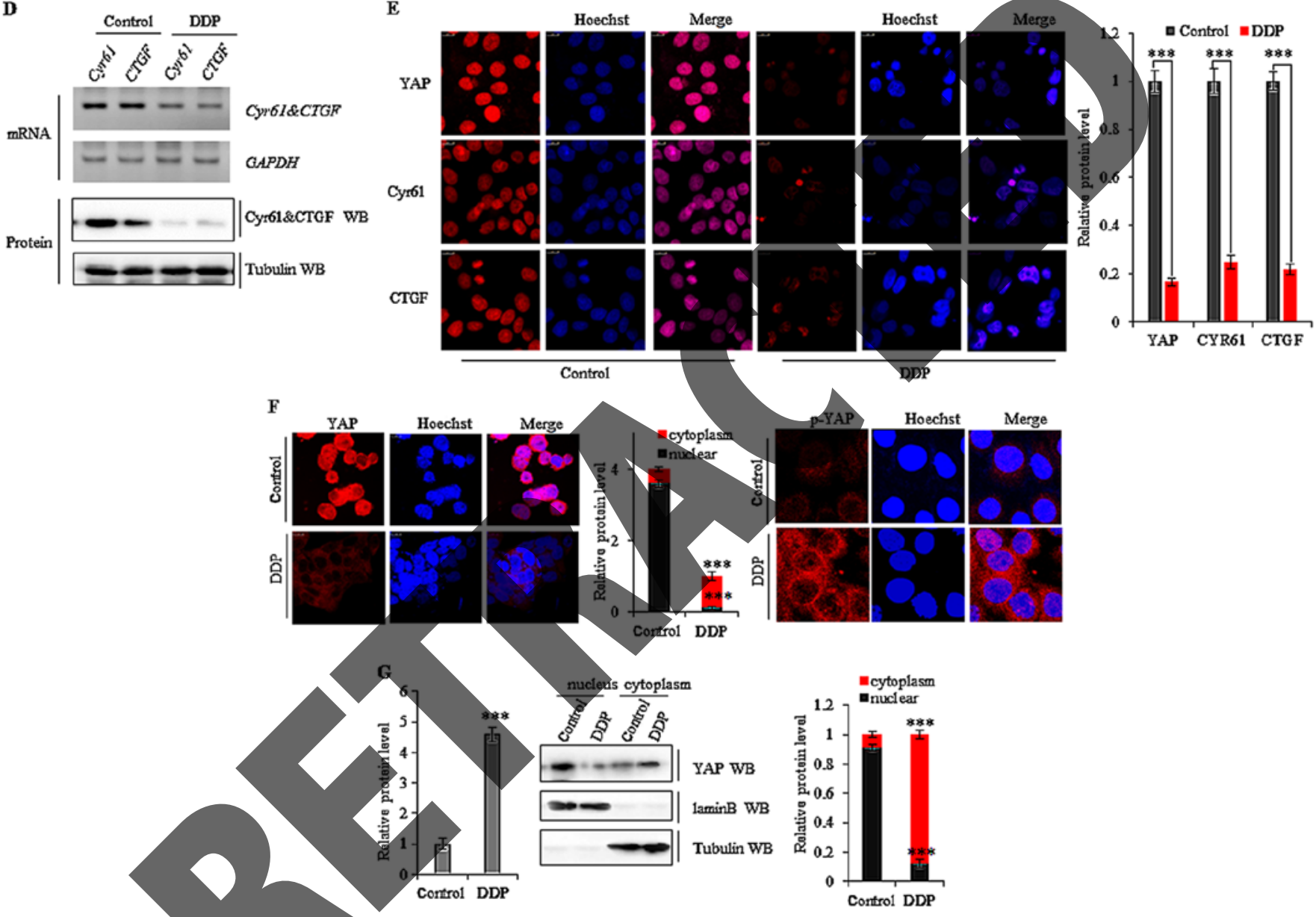

Figure 3. DDP reduces the activity of YAP in colon tumor cells. (A and B) RT-PCR and immunoblotting assays demonstrated that DDP treatment decreased YAP mRNA and YAP protein levels in SW620 cells at the indicated concentrations and time-points. (C) The effect of DDP on the luciferase activity of the YAP promoter in a luciferase reporter assay using SW620 and LoVo cells. (D) Gel-based RT-PCR and western blot analysis demonstrated a decrease in the mRNA and protein expression levels of YAP, CYR61 and CTGF in SW620 cells treated with DDP relative to control cells. (E) Immunofluorescent staining of YAP, CYR61 and CTGF protein showing decreased expression after treatment with DDP in SW620 cells compared to controls. (F) YAP and p-YAP immunofluorescent staining demonstrated that the translocation of YAP from the nucleus to the cytoplasm and p-YAP were enhanced after treatment with $6 \mu \mathrm{g} / \mathrm{ml}$ DDP for $36 \mathrm{~h}$ in SW620 cells. (G) Western blot analysis demonstrated that treatment with $6 \mu \mathrm{g} / \mathrm{ml}$ DDP for $36 \mathrm{~h}$ decreased YAP localization in the nucleus and enriched YAP localization in the cytoplasm of SW620 cells. Results were presented as mean \pm SD, and the error bars represent the SD of three independent experiments. ${ }^{*} \mathrm{P}<0.05 ;{ }^{* *} \mathrm{P}<0.01 ;{ }^{* * * *} \mathrm{P}<0.001$ vs. control group. DDP, cisplatin.

Cellular invasion is one of the most important defining features of cancer cells. Using a scratch assay, we found that treatment with $10 \mu \mathrm{g} / \mathrm{ml}$ DDP for $36 \mathrm{~h}$ decreased the YAP-induced migration in SW620 cells (Fig. 6C). Additionally, a Matrigel invasion and migration assay was performed to explore the effects of DDP on these properties in SW620 colon cancer cells. This indicated that treatment with $10 \mu \mathrm{g} / \mathrm{ml}$ DDP for $72 \mathrm{~h}$ decreased cell invasion and migration. DDP-induced inhibition of migration and growth was also partially reduced by overexpressing YAP in SW620 cells (Fig. 6D). As YAP-mediated invasion and migration could be inhibited by treatment with DDP in colon cancer cells, we next determined whether DDP influenced the YAP-mediated cellular phenotype transformation in colon cancer cells by examining markers 

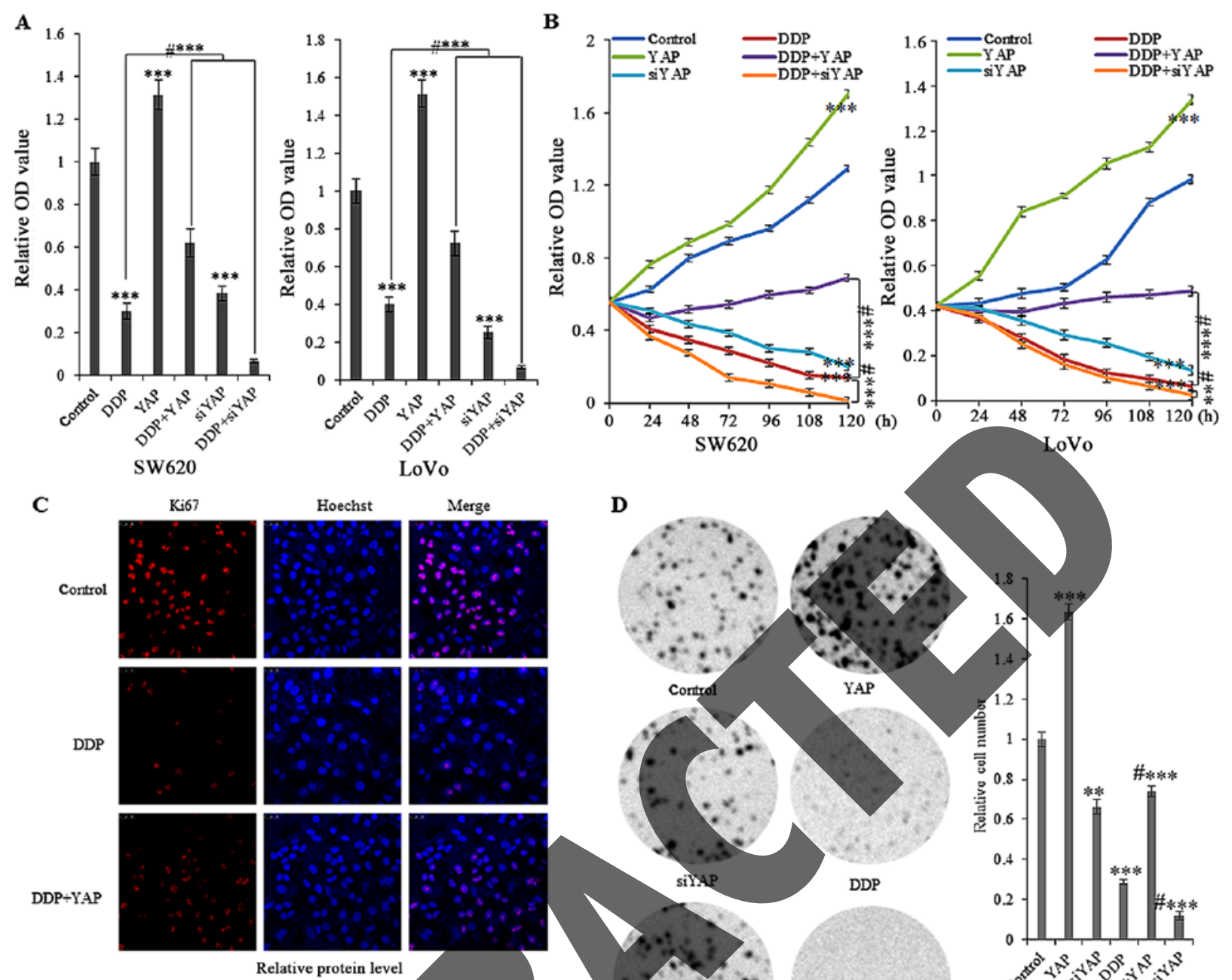

\section{D}
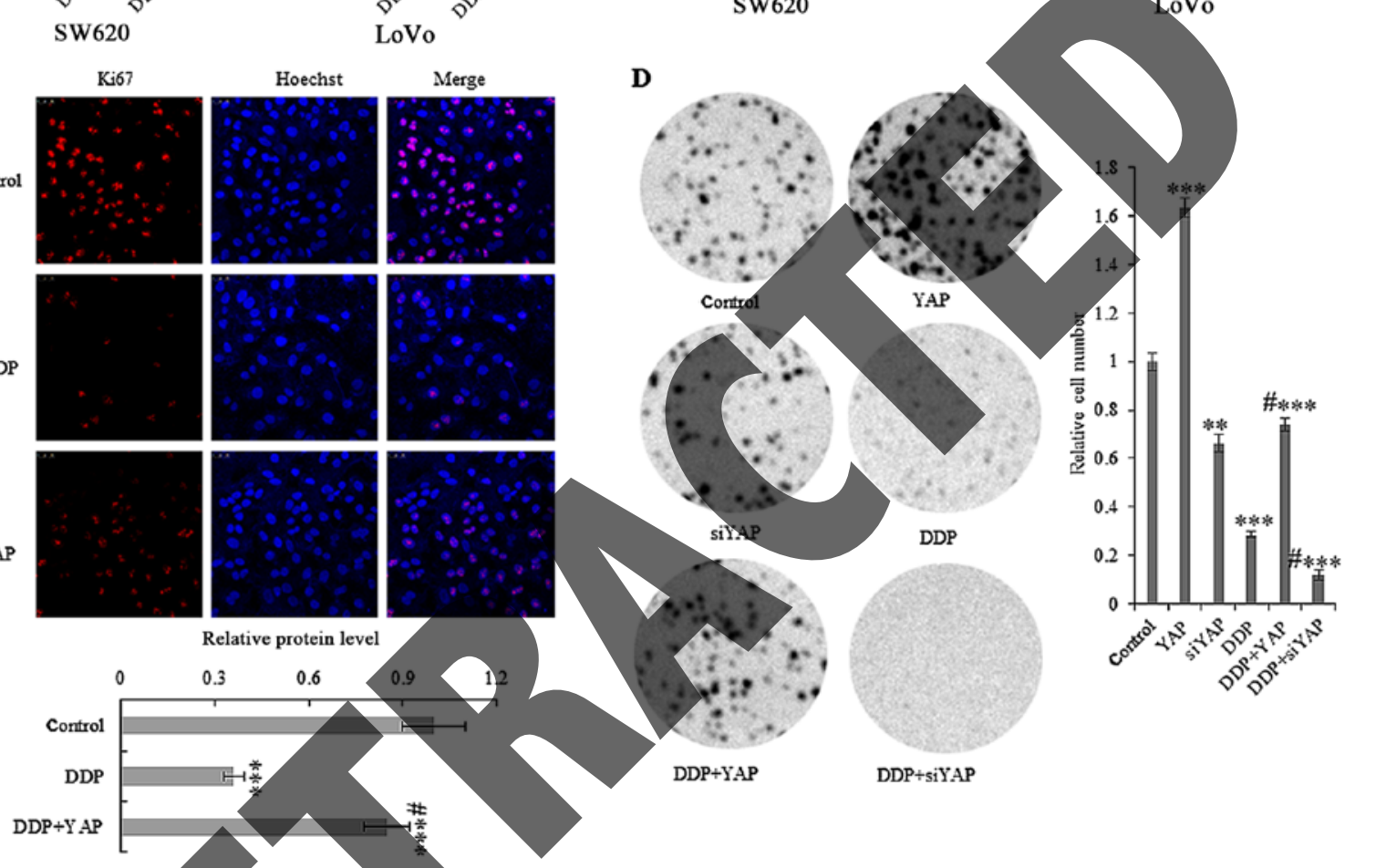

Figure 4. DDP affects YAP-mediated colon cell proliferation. (A) In vitro proliferation assays and (B) cell growth assays demonstrated that cells stably overexpressing YAP showed increased cellularproliferation in SW620 and LoVo cells after treatment with DDP had blocked YAP-induced cellular proliferation. YAP knockdown cells showed arrested cellular proliferation. (C) Immunofluorescent staining of Ki-67 protein demonstrated decreased proliferation after treatment with DDP in SW620 cells. (D) Colony formation was increased in stably overexpressing YAP cells and decreased in YAP-knockdown cells. However, DDP partially reduced YAP-induced cellular colony formation in SW620 cells. Results were presented as mean \pm SD, and the error bars represent the $\mathrm{SD}$ of three independent experiments. ${ }^{* *} \mathrm{P}<0.01 ;{ }^{* * * *} \mathrm{P}<0.001$ vs. control group and ${ }^{\# * * *} \mathrm{P}<0.01 ;{ }^{\# * * *} \mathrm{P}<0.001$ vs. co-treatment. DDP, cisplatin.

associated with EMT, such as vimentin and E-cadherin. As dislpayed in Fig. 6E and F, the mRNA and protein levels of E-cadherin were increased, whereas vimentin expression was decreased, after treatment with DDP for $72 \mathrm{~h}$ in SW620 cells. Ectopic overexpression of YAP decreased the DDP-induced expression changes in E-cadherin and vimentin expression, while an opposing effect was observed in YAP knockdown cells. This indicated that DDP interfered with the YAP-mediated EMT in colon tumor cells. Furthermore, immunofluorescent staining of E-cadherin confirmed that DDP increased E-cadherin protein expression under ectopic overexpression of YAP, while knockdown of YAP using siRNA partially blocked the DDP-induced increase in E-cadherin expression (Fig. 6G).

DDP inhibits the in vivo growth of in situ colon xenografts. To definitively address the central question as to whether DDP treatment can delay tumorigenesis in colon cancer, we explored the in vivo antitumor activity of DDP in colon cells using in situ colon xenografts and survival analysis. Approximately two weeks after the subcutaneous xenografting of SW620 cells into a concave niche of the ceca of mice, larger tumors were observed in the control group treated with PBS when compared to the group treated with DDP (Fig. 7A and B). Histochemical analysis of the tumors also indicated that tumor growth was inhibited by treatment with DDP relative to PBS. Further semi-quantitative immunohistochemical analysis of the YAP expression in the xenografts revealed that DDP treatment led to less YAP protein compared to the control group (Fig. 7C). Additional examination of the in situ xenografts by optical microscopy under H\&E staining indicated that several critical organs outside of the xenograft site, including the large intestine, small intestine, stomach, heart, liver, spleen, lungs 
$\mathbf{A}$

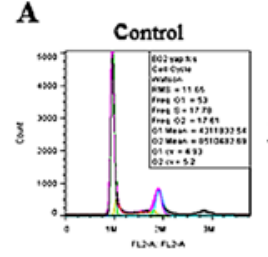

S1YAP
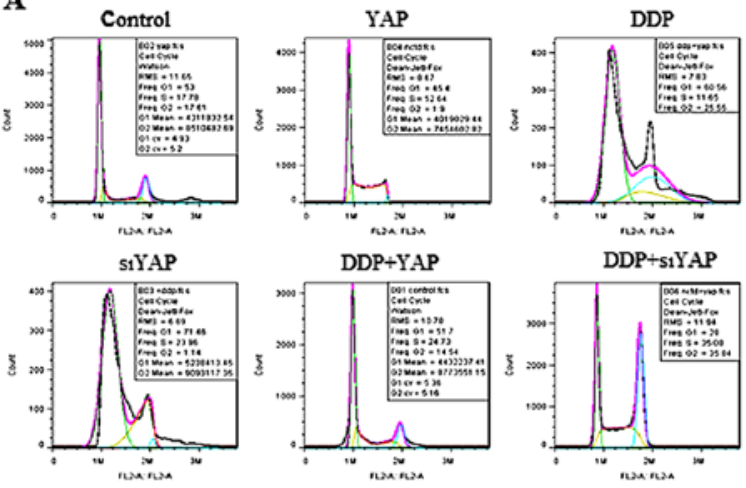

B
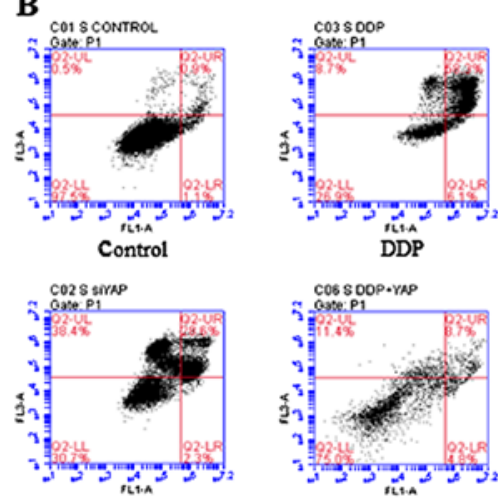

S1YAP

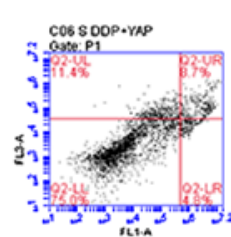

DDP+YAP

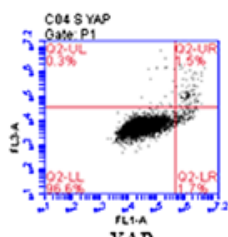

YAP

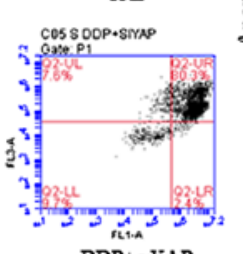

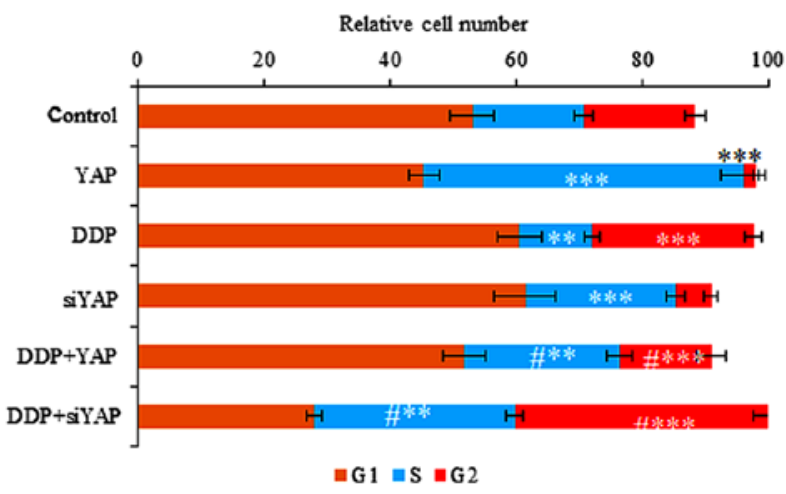

D

Control
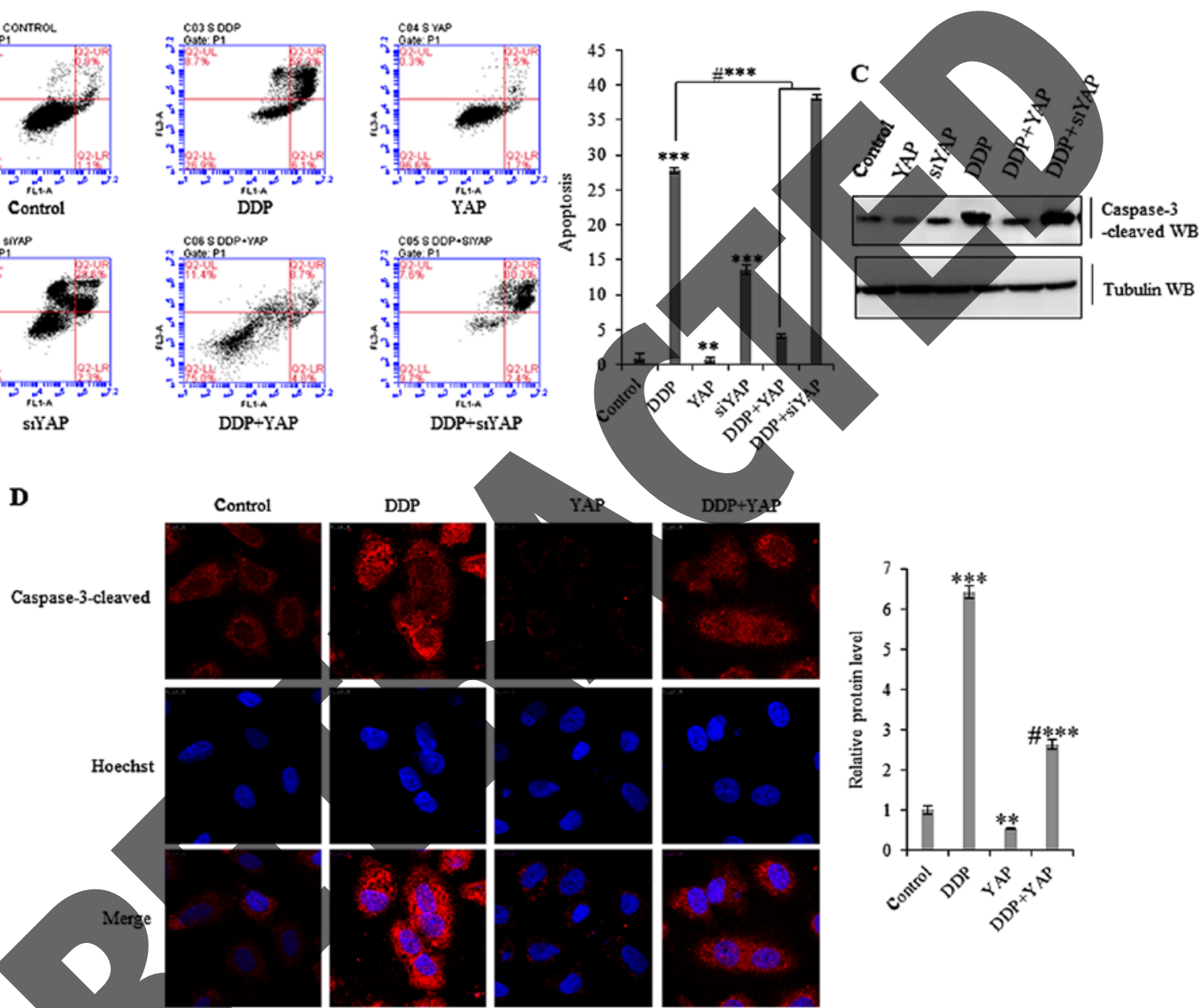

Figure 5. DDP affects the cell cycle and induces apoptosis in SW620 cells. (A) Cell cycle analysis revealed that treatment with $10 \mu \mathrm{g} / \mathrm{ml}$ DDP for $72 \mathrm{~h}$ induced an increase in the proportion of SW620 cells arrested in G2 phase, although YAP overexpression and knockdown of YAP either partially blocked or promoted this effect, respectively. (B) Annexin V staining and flow cytometry demonstrated that apoptosis was significantly decreased in SW620 cells after treatment with $10 \mu \mathrm{g} / \mathrm{ml}$ DDP for $72 \mathrm{~h}$. Ectopic overexpression of YAP blocked DDP-induced apoptosis, while knockdown of YAP using shRNA partially promoted apoptosis. (C) Immunoblotting assays demonstrated increased cleaved caspase-3 protein levels after treatment with DDP compared with SW620 control cells. Ectopic expression of YAP partially blocked DDP-induced caspase-3 expression, while knockdown of YAP using shRNA promoted caspase-3 expression. ${ }^{* *} \mathrm{P}<0.001,{ }^{* * *} \mathrm{P}<0.0001$ by Student's t-test. (D) Immunofluorescent staining of cleaved caspase- 3 confirmed that DDP increased the levels of this protein, while ectopic expression of YAP partially blocked the DDP-induced caspase-3 expression. Results were presented as mean \pm SD, and the error bars represent the SD of three independent experiments. ${ }^{* *} \mathrm{P}<0.01 ;{ }^{* * *} \mathrm{P}<0.001$ vs. control group and ${ }^{\# * * *} \mathrm{P}<0.01 ;{ }^{\# * * * *} \mathrm{P}<0.001$ vs. co-treatment. DDP, cisplatin.

and kidneys, were also severely damaged (Fig. 7D). We also confirmed that there was reduced body weight loss in mice treated with $1.5 \mathrm{mg} / \mathrm{kg}$ DDP during the treatment period compared to those administered PBS (Fig. 7E). Finally, it was observed that the survival times of the DDP treated group of xenograft mice were significantly longer than those of the control group (Fig. 7F).

\section{Discussion}

In the present study we have explored the efficacy and specificity of DDP in inhibiting colon carcinoma progression. Our data demonstrated that DDP is a suitable therapeutic drug candidate for colon cancers which sensitively and specifically targets the Mst/Yap signaling pathway. Although numerous 
A

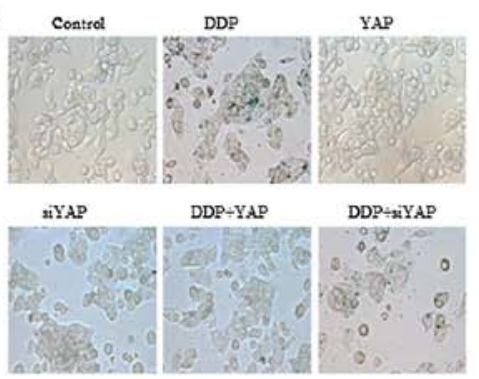

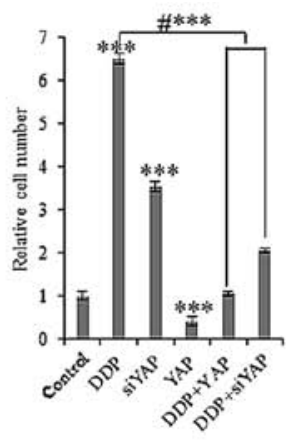

B
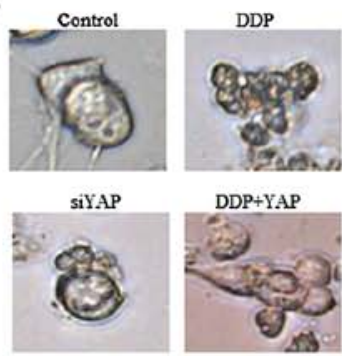

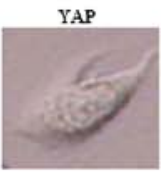

DDP+siYAP

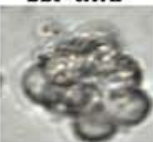

C
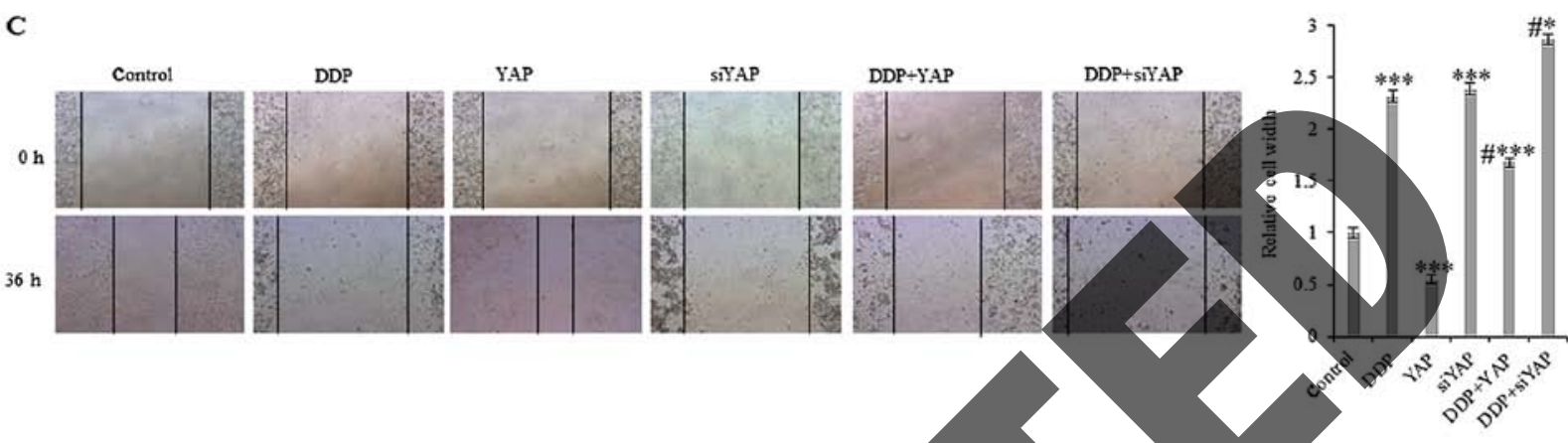

D

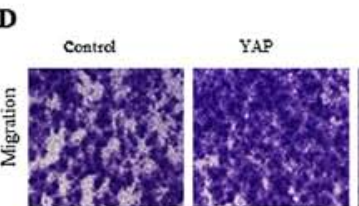

DDP siYAP
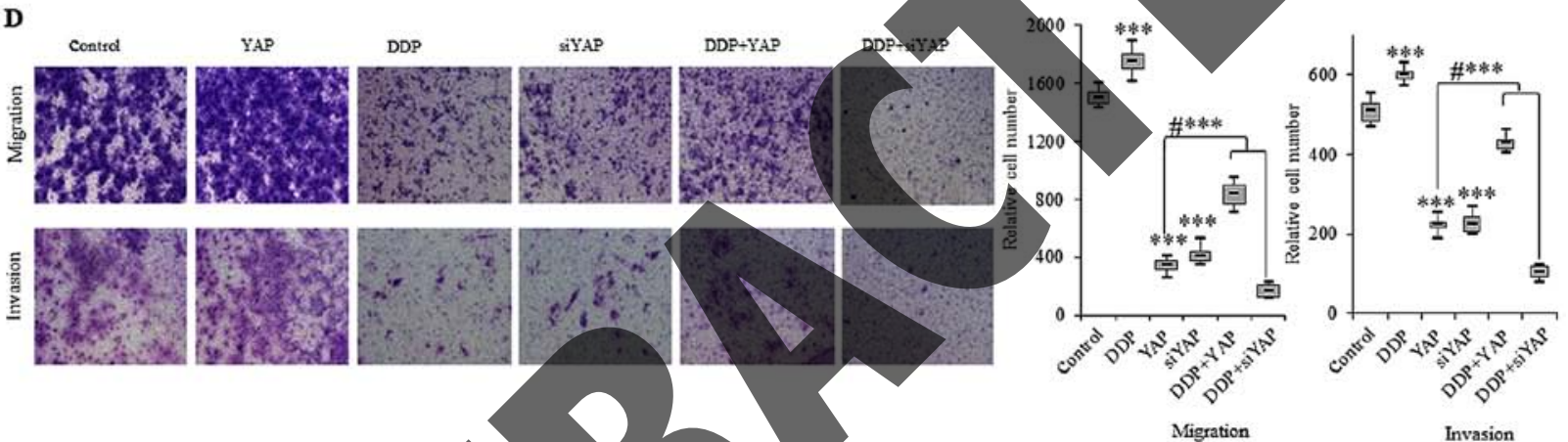

DDP+YAP
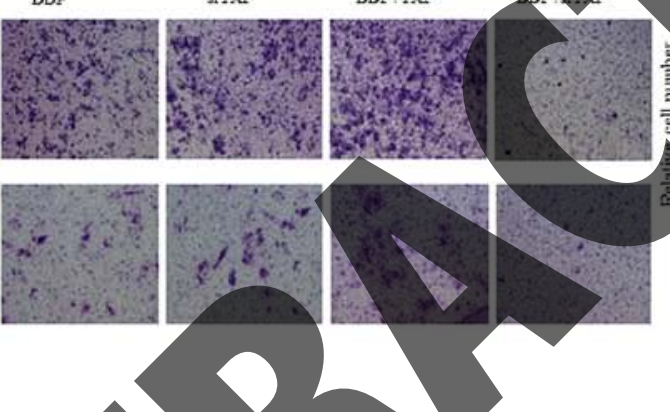

Migration

Invasion
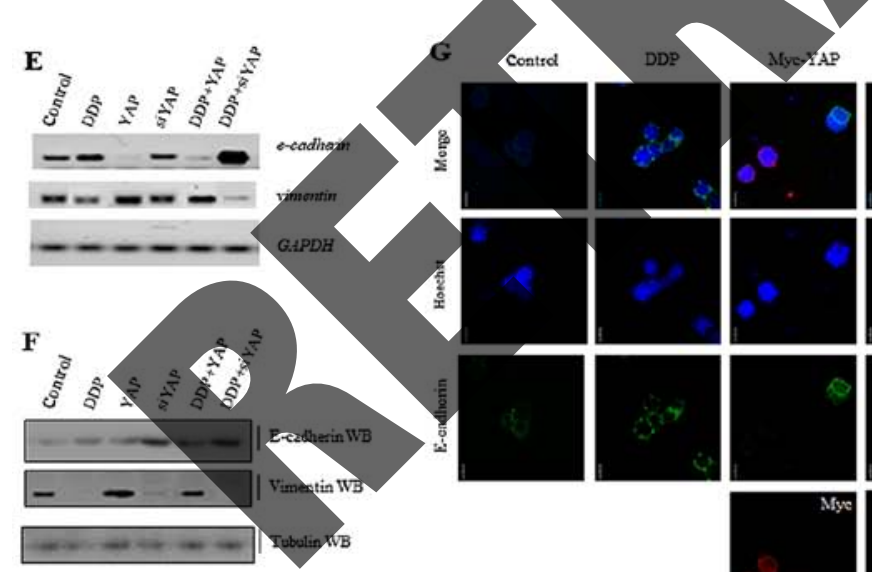

siYAP

DDP $\div$ MycYAP DDP $\div$ IYYAP
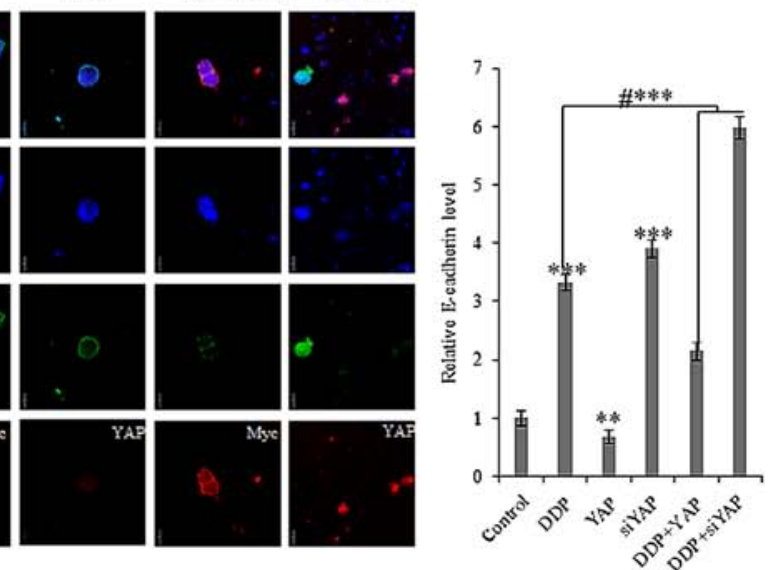

Figure 6. DDP affects YAP-mediated colon cancer cell senescence, invasiveness and EMT. (A) SA- $\beta$-Gal assays indicated that cell senescence was increased after treatment with $10 \mu \mathrm{g} / \mathrm{ml}$ DDP for $72 \mathrm{~h}$ in SW620 cells. Cells stably overexpressing YAP partially blocked senescence, while YAP-knockdown cells showed increased DDP-induced senescence. (B) Phase contrast microscopy exhibiting cellular morphology was markedly altered after treatment with $10 \mu \mathrm{g} / \mathrm{ml}$ DDP for $72 \mathrm{~h}$ in cells with or without stable overexpression of YAP and in cells with knockdown of YAP using shRNA. (C) Scratch assays indicated that the YAP-induced increase in cell migration was decreased after treatment with $10 \mu \mathrm{g} / \mathrm{ml}$ DDP for $36 \mathrm{~h}$ in SW620 cells. (D) Transwell assays showed that exposure to $10 \mu \mathrm{g} / \mathrm{ml}$ DDP for $72 \mathrm{~h}$ decreased cell invasion and migration in SW620 cells compared to control cells. Stably overexpressing YAP cells demonstrated partially blocked DDP-induced cellular invasive growth and migration, while YAP knockdown cells showed increased cellular invasive growth and migration. (E and F) RT-PCR and immunoblotting analysis revealed that the mRNA and protein levels of E-cadherin were significantly increased, whereas vimentin expression was decreased, in SW620 cells after treatment with DDP at $10 \mu \mathrm{g} / \mathrm{ml}$ for $72 \mathrm{~h}$. Stably overexpressing YAP cells partially blocked these DDP-induced expression changes in E-cadherin and vimentin expression, while cells with knockdown of YAP using shRNA exhibited a reverse effect. (G) Immunofluorescent staining of E-cadherin protein showing increased expression after treatment with $10 \mu \mathrm{g} / \mathrm{ml} \mathrm{DDP}$ for $72 \mathrm{~h}$ compared to control cells. Stably overexpressing YAP cells partially blocked DDP-induced expression of E-cadherin, while YAP knockdown cells promoted the expression of E-cadherin. Results were presented as mean $\pm \mathrm{SD}$, and the error bars represent the $\mathrm{SD}$ of three independent experiments. ${ }^{* *} \mathrm{P}<0.01 ;{ }^{* * * *} \mathrm{P}<0.001 \mathrm{vs}$. control group and ${ }^{* *} \mathrm{P}<0.05 ;{ }^{* * * *} \mathrm{P}<0.001$ vs. co-treatment. DDP, cisplatin. 


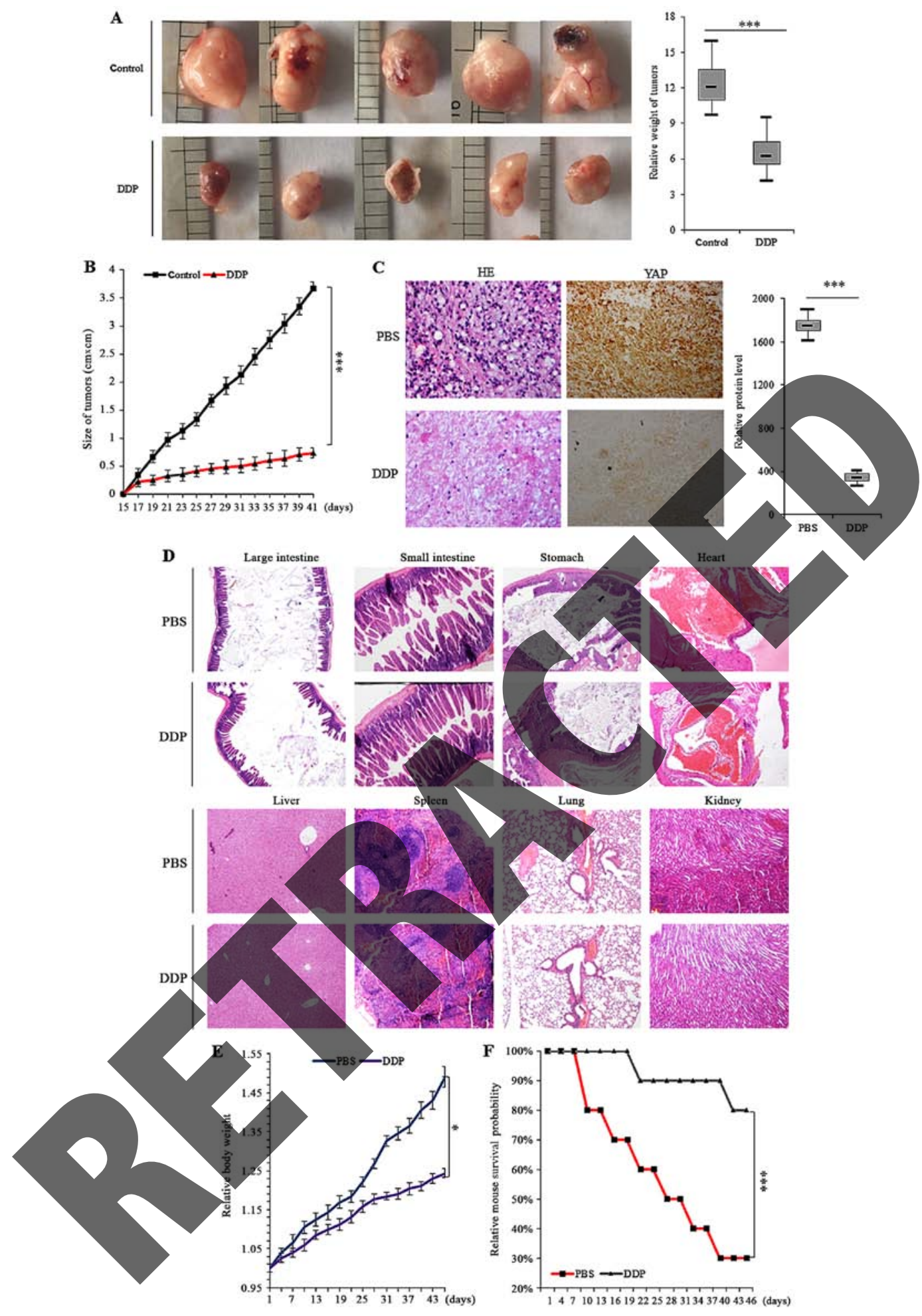

Figure 7. DDP inhibits the growth of in situ colon xenografts in vivo. (A) Xenograft mice with colon cancer cell tumors were treated with control (PBS) or DDP and the dimensions and weights of tumors were assessed at regular time-points. (B) Overall tumor sizes and growth curves. (C) H\&E microscopy of colon tumor nodules from primary SW620 cells in the subcutaneous xenografts of nude mice treated with control (PBS) and DDP. Immunohistochemical staining revealed that YAP expression was decreased in xenograft tumor tissues after treatment with DDP relative to PBS treatment. (D) Representative H\&E stained microscopic images of the large intestine, small intestine, stomach, heart, liver, spleen, colon and kidneys of nude mice with xenografted colon cancer cell tumors. There was less obvious organ damage in DDP-treated mice compared to PBS-treated mice. (E) The body weights of nude mice after treatment with DDP compared to PBS-treated mice demonstrated that DDP treatment did not significantly alter the body weight of animals and could also alleviate body weight loss. (F) KaplanMeier OS curves of mice treated with PBS and DDP. Results were presented as mean \pm SD, and the error bars represent the SD of three independent experiments. ${ }^{*} \mathrm{P}<0.05 ;{ }^{* * *} \mathrm{P}<0.001$ vs. control group. DDP, cisplatin; OS, overall survival; H\&E, hematoxylin and eosin; PBS, phosphate-buffered saline.

effective treatments and detection methods for colon tumors have improved survival rates for patients, the disease remains one of the most aggressive malignant cancers and has a particularly high fatality rate. Due to the pathology of the disease, most patients present in advanced stages, meaning that the best treatment options are palliative, such as radiotherapy 
and chemotherapy. However, normal tissue cells are often destroyed by these indiscriminate methods of treatment. Therefore, identifying novel natural compounds that are highly selective for cancer cells and have low non-specific toxicity, is important for improving cancer therapy.

The anticancer activity of DDP, a commonly used chemotherapeutic agent developed in China, has been examined in several studies. Unlike many other treatments, DDP induces few side-effects in the digestive system and is also relatively easy to synthesize. It has been demonstrated to inhibit cell growth in several cancer cell lines and transplanted tumors, while simultaneously increasing white blood cell counts by sensitizing the bone marrow. Due to this effect, the drug has also some antagonistic effects against leukopenia. In addition, clinical approaches that apply DDP as a mono-therapeutic agent have reported that the drug has beneficial effects in patients with several different types of tumors simultaneously. Our data indicated that DDP inhibited the in vitro growth of SW620 and LoVo human colon cancer cell lines in a dose- and time-dependent manner. Immunoblotting analysis revealed that DDP dose-dependently downregulated YAP and the expression of downstream target genes CTGF and CYR61 via an interaction with the promoter region of the $Y A P$ gene, indicating a functional mechanism. This downregulation of downstream genes led to cell cycle arrest in colon cancer cells and the observed inhibition of tumor growth. Notably, DDP also suppressed SW620 cell invasion and migration without reducing cell viability. These data indicated that DDP may have wider direct or adjuvant therapeutic applications for the treatment of human colon tumors and this will require further examination.

Currently, the efficacy of available colon cancer therapeutic options is limited by the development of treatment resistance in cancer cells. YAP in particular is a novel anticancer drug target gene that has been associated with high chemoresistance in colo cancer. Aberrant activation of this oncogene contributes to the initiation, progression and metastasis of colon tumors and associates with poor prognosis. This would also act to promote drug resistance against the targeted therapy. YAP has been found to be highly activated in colon cancer and reducing its expression in cancer cells may inerease the therapeutic effects of colon cancer treatments. Our results demonstrated that the Mst/Yap signaling pathway was abnormally activated in colon cancer tumor tissues and cells. Like other tumors, this would lead to increased cell growth and invasiveness. However, our functional data demonstrated that DDP specifically suppressed the Mst/Yap signaling pathway, affecting YAP-mediated colon cancer progression and metastasis, by arresting the cell cycle and inducing apoptosis and cell senescence. We also confirmed that DDP suppression of YAP activity reduced EMT and decreased the motility, metastatic and invasive capacities of colon cancer cells in vitro, probably by enhancing the expression of E-cadherin and decreasing the expression of fibronectin/vimentin.

In addition, our data demonstrated that DDP not only regulated $Y A P$ transcriptional activity, but also post-translationally modified YAP, affecting the subcellular distribution of phosphorylated and unphosphorylated protein between the cytosol and the nucleus. Markedly, DDP demonstrated no kinase activity, but some other researches revealed that zyxin formed a ternary complex with Siah2 and Lats2 responding to TGF- $\beta$ stimuli, thus stabilized their interaction and facilitated deactivation of the YAP signaling pathway, thereby promoting tumor progression $(33,34)$. Furthermore, DDP could regulate the activity of TGF- $\beta$ to suppressed lung cancer progression and metastasis (35). Therefore, we hypothesized that DDP may regulate cytoplasmic $\mathrm{p}-\mathrm{YAP}$ by regulating the upstream signals of YAP, however we should further explore this hypothesis. Furthermore, we have shown that DDP dose-dependently and time-dependently increased p-YAP alongside decreasing YAP mRNA and YAP protein expression in SW620 colon cancer cells. This provided further information to elucidate the molecular mechanisms of DDP and the therapeutic activity of the drug against colon cancer. However, it is important to note that this study is prospective and, although these are important results, there are many issues that require further examination. These include understanding the mechanism through which the inhibition of YAP expression by DDP improves the chemotherapeutic treatment of drug-resistant colon carcinomas.

In conclusion, the present study indicated that the anticancer drug DDP was an effective therapeutic drug for the treatment of human colon cancer. DDP enhanced apoptosis, senescence and cell cycle arrest in cancer cells by suppressing YAP expression, thereby inhibiting cell proliferation. Notably, we demonstrated that the effects of DDP were potentially mediated via an interaction with the promoter region of the $Y A P$ gene. Our results indicated that DDP acted as a chemotherapeutic antitumor agent that can be used for suppressing tumorigenesis and the initiation of colon tumor via the downregulation of YAP in human colon cancer. The inhibition of cancer cell proliferation by DDP was associated with interference in cell cycle progression in colon tumor cells, increasing the proportion of cells in the $\mathrm{G} 2 / \mathrm{M}$ phase. These data improved our understanding of colon cancer and the role that the Mst/Yap pathway plays in ancer progression. In addition, the present indicated that DDP may serve as an important future treatment for colon cancer.

\section{Acknowledgements}

Not applicable.

\section{Funding}

The present study was supported by the Science and Technology Development Foundation of Yantai (no. 2015ZH082), the Natural Science Foundation of Shandong Province (nos. ZR2016HB55 and ZR2017PH067) and the Research Foundation of Binzhou Medical University (nos. BY2015KYQD29 and BY2015KJ14).

\section{Availability of data and materials}

The datasets used during the present study are available from the corresponding author upon reasonable request.

\section{Authors' contributions}

JG, KL and LC designed the experiments. KL, JG, YW, DJ and $\mathrm{HJ}$ performed the work. JG, KL and LC analyzed the data and compiled the figures. JG and LC wrote the manuscript. All authors read and approved the manuscript and agreed to be accountable for all aspects of the research in ensuring that the 
accuracy or integrity of any part of the work are appropriately investigated and resolved.

\section{Ethics approval and consent to participate}

The experimental protocol was approved by the Research Ethics Committee of Binzhou Medical University (approval no. 2017-014-01 for the human tissues and 2017-05-02 for the mouse tissues). Written informed consents were obtained from all patients.

\section{Consent for publication}

Not applicable.

\section{Competing interests}

The authors declare that they have no competing interests.

\section{References}

1. Xu Z, Jiang H, Zhu Y, Wang H, Jiang J, Chen L, Xu W, Hu T and Cho $\mathrm{CH}$ : Cryptotanshinone induces ROS-dependent autophagy in multidrug-resistant colon cancer cells. Chem Biol Interact 273: 48-55, 2017.

2. Udawat H, Nunia V, Mathur P, Udawat HP, Gaur KL, Saxena AK and Mohan MK: Histopathological and immunohistochemical findings in congenital pouch colon: A prospective study. Pathobiology 84: 202-209, 2017.

3. Senol S, Ceyran AB, Kösemetin D, Gobanoglu B, Ay Duran EA and Leblebici M: Immunohistochemical profile tumor pathways and prognostic significance in colon aden cinomas. J Environ Pathol Toxicol Oncol 36: 29-41, 2017.

4. Hakoda K, Yoshimitsu M, Emi M, Omori Kaneko M, Ohdan H and Hirabayashi N: Complete pathologica response of multiple huge liver metastases of colon cancer: A case report. Oxf Med Case Reports 2017: omx016, 2017.

5. Sloothaak DAM, van der Linden RLA, Bemelman WA, Lips DJ, van der Lind

Tanis PJ, Bosscha K, van der Za cations of occult nodal tumour cells in stage I and II colon cancer: The correlation between micrometastasis and disease recurrence. Eur J Surg Oncol

6. Nakamura Y, Hattori N, Tida N, Yamashita S, Mori A, Kimura K, Yoshino T and Ushijima T: Targeting of super-ephancers and mutant BRAF can suppress growth of BRAF-mutant colon cancer cells via repression of MAPK signaling pathway. Cancer Lett 402: 100-109.

7. Guo J, Wu Y, Yang L, Du J, Gong K, Chen W, Dai J, Li X and Xi S: Repression of YAP by NCTD disrupts NSCLC progression. Oncotarget 8

8. Azzolin L, Panciera T, Soligo S, Enzo E, Bicciato S, Dupont S, Bresolin S, Frasson C, Basso G, Guzzardo V, et al: YAP/TAZ incorporation in the $\beta$-catenin destruction complex orchestrates the Wnt response. Cell 158: 157-170, 2014.

9. Martin K, Pritchett J, Llewellyn J, Mullan AF, Athwal VS, Dobie R, Harvey E, Zeef L, Farrow S, Streuli C, et al: PAK proteins and YAP-1 signalling downstream of integrin beta-1 in myofibroblasts promote liver fibrosis. Nat Commun 7: 12502, 2016.

10. Moleirinho S, Hoxha S, Mandati V, Curtale G, Troutman S, Ehmer U and Kissil JL: Regulation of localization and function of the transcriptional co-activator YAP by angiomotin. eLife 6: e23966, 2017.

11. TranchantR, Quetel L, Tallet A, Meiller C, Renier A, de Koning L, de Reynies A, Le Pimpec-Barthes F, Zucman-Rossi J, Jaurand MC, et al: Co-occurring mutations of tumor suppressor genes, LATS2 and NF2, in malignant pleural mesothelioma. Clin Cancer Res 23: 3191-3202, 2017.

12. Feng G, Zhu Z, Li WJ, Lin Q, Chai Y, Dong MQ and Ou G: Hippo kinases maintain polarity during directional cell migration in Caenorhabditis elegans. EMBO J 36: 334-345, 2017.

13. Wang L, Luo JY, Li B, Tian XY, Chen LJ, Huang Y, Liu J, Deng D, Lau CW, Wan S, et al: Integrin-YAP/TAZ-JNK cascade mediates atheroprotective effect of unidirectional shear flow. Nature 12: 7, 2016.
14. Moroishi T, Hayashi T, Pan WW, Fujita Y, Holt MV, Qin J, Carson DA and Guan KL: The Hippo pathway kinases LATS1/2 suppress cancer immunity. Cell 167: 1525-1539.e17, 2016.

15. Sharif GM and Wellstein A: Cell density regulates cancer metastasis via the Hippo pathway. Future Oncol 11: 3253-3260, 2015

16. Kim T, Hwang D, Lee D, Kim JH, Kim SY and Lim DS: MRTF potentiates TEAD-YAP transcriptional activity causing metastasis. EMBO J 36: 520-535, 2017.

17. Yamamoto M, Ohsawa S, Kunimasa $\mathrm{K}$ and Igaki T: The ligand Sas and its receptor PTP10D drive tumour-suppressive cell competition. Nature 542: 246-250, 2017.

18. Jiao S, Li C, Hao Q, Miao H, Zhang L, Li L and Zhou Z: VGLL4 targets a TCF4-TEAD4 complex to coregulate Wnt and Hippo signalling in colorectal cancer. Nat Commun 8: 14058, 2017.

19. Oun R and Rowan E: Cisplatin induced arrhythmia; electrolyte imbalance or disturbance of the SA node? Eur J Pharmacol 811: 125-128, 2017.

20. Alterio D, Cossu Rocca M, Russell-Edu W, Dicuonzo S, Fanetti G, Marvaso G, Preda L, Zorzi S, Verri E, Nole' F, et al: Med Oncol 34: 86, 2017.

21. Zhang F, Yu X, Liu X, Zhou T, Nie T, Cheng M, Liu H, Dai M and Zhang B: ABT-737 potentiates cisplatin-induced apoptosis in human osteosarcoma cells via the mitochondrial apoptotic pathway. Oncol Rep 38:2301-2308, 2017.

22. Wang J, Kho DH, Zhou JY, Davis RJ and Wu GS: MKP-1 suppresses PARP-1 degradation to mediate cisplatin resistance. Oncogene 36. 5939-5947, 2017.

23. Chen LG, Xia YJ and Cui Y: Upregulation of miR-101 enhances the cytotoxic effect of anticancer drugs through inhibition of colon cancer cell proliferation. Oncol Rep 38: 100-108, 2017.

24. Xu M, Tang X, Guo J, Sun W and Tang F: Reversal effect of adenovirus-mediated human interleukin 24 transfection on the cisplatin resistance of A549/DDP lung cancer cells. Oncol Rep 38: 2843-2851, 2017.

25. Jacobs J, Deschoolmeester V, Rolfo C, Zwaenepoel K, an den Bossche J, Deben C, Silence K, de Haard H, Hermans C, Rottey S, et al: Preclinical data on the combination of cisplatin and anti-CD 70 therapy in non-small cell lung cancer as an excellent match in the era of combination therapy. Oncotarget 8: 74058-74067, 2017.

26. Mukherjee S, Dash S, Lohitesh K and Chowdhury R: The dynamic role of autophagy and MAPK signaling in determining cell fate under cisplatin stress in osteosarcoma cells. PLoS One 12: e0179203, 2017.

. Li L, Duan W, Zhang L, Li X, Fu X, Wang X, Wu J, Sun Z, Zhang X, Chang Y, et al: The efficacy and safety of gemcitabine, cisplatin, prednisone, thalidomide versus CHOP in patients with newly diagnosed peripheral T-cell lymphoma with analysis of biomarkers. Br J Haematol 178: 772-780, 2017.

28. Choi BY, Joo JC, Lee YK, Jang IS, Park SJ and Park YJ: Anticancer effect of Scutellaria baicalensis in combination with cisplatin in human ovarian cancer cell. BMC Complement Altern Med 17: 277, 2017.

29. Rudolph C, Melau C, Nielsen JE, Vile Jensen K, Liu D, Pena-Diaz J, Rajpert-De Meyts E, Rasmussen LJ and Jørgensen A: Involvement of the DNA mismatch repair system in cisplatin sensitivity of testicular germ cell tumours. Cell Oncol (Dordr) 40: 341-355, 2017.

30. Ge L, Li DS, Chen F, Feng JD, Li B and Wang TJ: TAZ overexpression is associated with epithelial-mesenchymal transition in cisplatin-resistant gastric cancer cells. Int J Oncol 51: 307-315, 2017.

31. Ekinci Akdemir FN, Albayrak M, Calik M, Bayir Y and Gulcin I: The protective effects of p-coumaric acid on acute liver and kidney damages induced by cisplatin. Biomedicines 5: E18, 2017.

32. Győrffy B, Surowiak P, Budczies J and Lánczky A: Online survival analysis software to assess the prognostic value of biomarkers using transcriptomic data in non-small-cell lung cancer. PLoS One 8: e82241, 2013.

33. Ma B, Cheng H, Gao R, Mu C, Chen L, Wu S, Chen Q and Zhu Y: Zyxin-Siah2-Lats2 axis mediates cooperation between Hippo and TGF- $\beta$ signalling pathways. Nat Commun 7: 11123, 2016.

34. Gaspar P, Holder MV, Aerne BL, Janody F and Tapon N: Zyxin antagonizes the FERM protein expanded to couple F-actin and Yorkie-dependent organ growth. Curr Biol 25: 679-689, 2015.

35. Wang J, Chen Y, Xiang F, Li M, Li H, Chi J and Ren K: Suppression of TGF- $\beta 1$ enhances chemosensitivity of cisplatinresistant lung cancer cells through the inhibition of drug-resistant proteins. Artif Cells Nanomed Biotechnol 8: 1-8, 2017.

This work is licensed under a Creative Commons Attribution-NonCommercial-NoDerivatives 4.0 International (CC BY-NC-ND 4.0) License. 